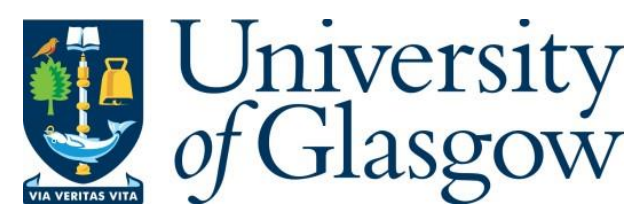

Zhang, L., Ijaz, A., Xiao, P., Quddus, A. and Tafazolli, R. (2017) Subband filtered multi-carrier systems for multi-service wireless communications. IEEE Transactions on Wireless Communications, 16(3), pp. 1893-1907.

There may be differences between this version and the published version. You are advised to consult the publisher's version if you wish to cite from it.

http://eprints.gla.ac.uk/143222/

Deposited on: 6 July 2017

Enlighten - Research publications by members of the University of Glasgow http://eprints.gla.ac.uk 


\title{
Subband Filtered Multi-carrier Systems for Multi-service Wireless Communications
}

\author{
Lei Zhang, Ayesha Ijaz, Pei Xiao, Atta Quddus and Rahim Tafazolli
}

\begin{abstract}
Flexibly supporting multiple services, each with different communication requirements and frame structure, has been identified as one of the most significant and promising characteristics of next generation and beyond wireless communication systems. However, integrating multiple frame structures with different subcarrier spacing in one radio carrier may result in significant inter-service-band-interference (ISBI). In this paper, a framework for multi-service (MS) systems is established based on subband filtered multi-carrier system. The subband filtering implementations and both asynchronous and generalized synchronous (GS) MS subband filtered multi-carrier (SFMC) systems have been proposed. Based on the GS-MS-SFMC system, the system model with ISBI is derived and a number of properties on ISBI are given. In addition, low-complexity ISBI cancelation algorithms are proposed by precoding the information symbols at the transmitter. For asynchronous MS-SFMC system in the presence of transceiver imperfections including carrier frequency offset, timing offset and phase noise, a complete analytical system model is established in terms of desired signal, intersymbol-interference, inter-carrier-interference, ISBI and noise. Thereafter, new channel equalization algorithms are proposed by considering the errors and imperfections. Numerical analysis shows that the analytical results match the simulation results, and the proposed ISBI cancelation and equalization algorithms can significantly improve the system performance in comparison with the existing algorithms.
\end{abstract}

Index Terms-multi-service multi-carrier system, inter-serviceband-interference, universal filtered multi-carrier, subband filtered multi-carrier, transceiver imperfection, generalized synchronization

\section{INTRODUCTION}

The next generation wireless communication (5G and beyond) is required to support a greater density of users, higher data throughput, ultra-high reliability and ultra-low latency communications to meet the vision of "everything everywhere and always connected" with "provision of perception of infinite capacity" [1], [2]. However, these multi-fold requirements are generally driven by different type of services and use cases, resulting in different optimal radio frame structure design criteria. For example, the service for machine type communications (MTC) might require smaller subcarrier spacing (thus larger symbol duration) to support massive delay-tolerant devices [2], [3]. Vehicle to vehicle (V2V) communications, on the other hand, have more stringent latency requirements, thus, symbol duration might be significantly reduced. The subcarrier spacing and symbol duration of high data rate transmission are, however, constrained by doubly-dispersive channel, i.e.,

The authors are with the 5G Innovation Centre (5GIC) and Institute for Communication Systems (ICS), University of Surrey, Guildford, GU2 7XH, UK (Email: lei.zhang@surrey.ac.uk). channel coherence time and coherence bandwidth. Therefore, there is a limit on subcarrier spacing and symbol duration in order to avoid performance bottlenecks by channel degradation. Hence, it is cumbersome to design a unified all-inone radio frame structure which meets these requirements for all types of services. In addition, separate radio designs for separate services make the operation and management of the system highly complex, expensive and spirally inefficient. One viable solution is to divide the system bandwidth into several service bands with each used for a different type of service [4]. Such a multi-service (MS) system is shown in Fig. 1, where a specifically optimized frame structure has been designed for different types of services, with or without a certain guard band (GB) between them to mitigate the interference.

However, combining multiple frame structures with different subcarrier spacings in one frequency band will destroy the orthogonality of a multi-carrier system, resulting in interservice-band-interference (ISBI). The interference level depends on the subcarrier spacing difference and the GB between the service bands. Moreover, the choice of waveform is also a key factor to determine the interference level. Orthogonal frequency division multiplexing (OFDM) has been used in several standardizations such as the 3rd Generation Partnership Project (3GPP) Long Term Evolution (LTE) and Institute of Electrical and Electronics Engineers (IEEE) 802.11. It is not suitable for this MS frame structure due to its high out of band (OoB) emission level. Several new waveforms are proposed to reduce OoB emission level for next generation communications, such as subband filtered multi-carrier (SFMC) based systems including universal filtered multi-carrier (UFMC) [5], [6], [7], [8], [9] and filtered OFDM (f-OFDM) [10], [4], filter bank multi-carrier (FBMC) [11], [12] and generalized frequency division multiplexing (GFDM) [13], [14]. Among these, SFMC based system has drawn significant attention from academia and industry in the last few years. It inherits the advantages of OFDM system such as low complexity and effective one-tap channel equalization [15], [16]. In addition, it also achieves comparable OoB emission as FBMC systems [5], [6], [7], [17]. Note that a fast-convolution implementation of multirate filter bank based waveform has been implemented and analyzed by transforming the filtering operation into the fast Fourier transform (FFT) domain [18], [19], with filtered cyclic prefix (CP) OFDM as a special case [20].

There are several methods to mitigate or eliminate the ISBI by using either radio frequency (RF) or baseband signal processing. Adopting RF filter and hardware components to isolate service bands can eliminate the interference. However, it comes at the expense of not only compromised accuracy and 


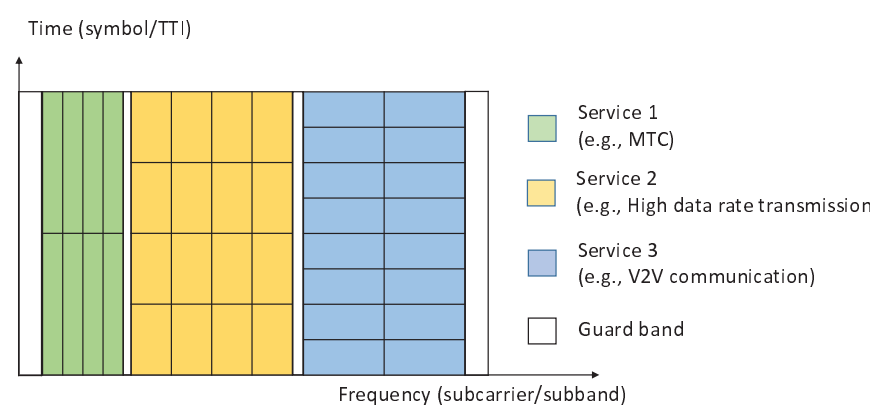

Fig. 1. An example of multi-service multi-carrier system.

increased system complexity but also reduction in spectrum efficiency. From the baseband processing perspective, using new waveforms and/or GB between service bands is an effective way to mitigate ISBI [4]. However, it also results in spectrum efficiency reduction. In this paper, we propose low-complexity baseband signal processing algorithms to precancel the ISBI by precoding the information symbols before transmission. This solution can effectively avoid the overhead problem of the two aforementioned methods.

Note that it has been shown via simulations in [4] that using a couple of subcarriers as GB between two service bands are sufficient for low and moderate modulation and coding schemes (MCS), based on the f-OFDM waveform, which is one of the SFMC based implementations with partly overlapping subband filtered OFDM symbols [10], [4]. However, to the best of the authors' knowledge, the generic system model and analytical results on ISBI for MS-SFMC systems are still open issues. In addition, it is not yet clear how the difference between subcarrier spacing and GB between service bands would affect the interference level. Based on our derived generic system model, all of the aforementioned questions will be properly answered in this paper.

The frequency resource structure in an MS system is more complex than the original single service system due to the introduction of the service band. The problem as to how the subband filtering is performed has not yet been systematically discussed in the literature. Differences in symbol durations (of different services) make the synchronization of the whole system unattractive. A new concept called generalized synchronized (GS) system will be proposed in this paper to address this problem and facilitate system implementation and analysis.

To provide useful frame structure design guidelines for next generation communication in practical environments, it is necessary to investigate the impacts of transceiver imperfections including carrier frequency offset (CFO), timing offset (TO) and phase noise (PN) on the performance. To do this, we establish a comprehensive analytical framework and system model by taking all of the aforementioned imperfections into consideration in multipath fading environments. This is one of the aims of this paper and this comprehensive analytical framework is also essential for ISBI pre-cancelation and channel equalization algorithms proposed in this paper.

The performance analysis and algorithm design for SFMC (mainly UFMC) system was focused on the single service case in the literature. In the presence of CFO, [17] analyzed the performance of single service UFMC systems and a filter was optimized to minimize the out of band leakage (OBL) in [21] by considering both $\mathrm{CFO}$ and $\mathrm{TO}$ for single-path flat fading channel. In [22], the authors proposed a comprehensive system model and performance analysis for single service in a multi-user UFMC system by considering transceiver imperfections and based on this model, filter length and ZP length optimizations were formulated.

The contributions and novelties of this paper are summarized as follows:

- We first build a framework for MS-SFMC system and categorize the possible subband filtering implementations and synchronized systems in frequency and time domains, respectively. Specifically, the physical resource block (PRB), user and service based subband filtering methods are defined in frequency domain. In the time domain, we define both non-synchronized and generalized synchronized MS systems. In the latter case, services have an integral least common multiplier (LCM) symbol, forming the basis of system modeling, performance analysis and ISBI cancelation algorithms of GS-MS system.

- Based on the GS system and one of the SFMC implementations, i.e, UFMC, and in the absence of transceiver imperfections and sufficient guard interval between symbols, we first establish a system model for MS-UFMC systems with arbitrary values of subcarrier spacing and GB. Then propositions are made on ISBI to explain why SFMC system is more robust to the ISBI than the OFDM system. These analytical results provide useful guidance for MS system radio frame structure design. In addition, we propose low complexity ISBI cancelation algorithms using the criteria of zero-forcing (ZF) and minimum mean square error (MMSE), respectively. The algorithms are flexible to support arbitrary bandwidth interference cancelation.

- In the presence of transceiver imperfections for asynchronous MS system, we derive an analytical expression for MS-UFMC system in terms of desired signal, inter-carrier interference (ICI), inter-symbol interference (ISI), ISBI and noise by considering CFO, TO, PN and insufficient ZP length between symbols. Based on the analytical framework, we propose channel equalization algorithms that can provide significant gain in terms of BER performance in comparison to OFDM and the stateof-the-art (SoTA) UFMC systems.

Notations: Vectors and matrices are denoted by lowercase and uppercase bold letters, and $\{\cdot\}^{H},\{\cdot\}^{T},\{\cdot\}^{*}$ stand for the Hermitian conjugate, transpose and conjugate operation, respectively. We use trace $\{\mathbf{A}\}$ and $\operatorname{diag}\{\mathbf{A}\}$ to denote the trace of matrix $\mathbf{A}$ and taking the diagonal matrix of $\mathbf{A}$ to reframe a diagonal matrix, respectively. $\mathbf{I}_{M}$ and $\mathbf{0}_{m \times n}$ refer to $M \times M$ identity matrix and $m \times n$ zero matrix, respectively. Operator $*$ denotes linear convolution of two vectors. $\mathcal{E}\{a\},\lceil a\rceil$ and $\lfloor a\rfloor$ denote the expectation, ceiling and floor operations on $a$, respectively. $\operatorname{Blkdiag}(\mathbf{A}, N)$ refers to a 
block diagonal matrix generated by $N$ repetitions of $\mathbf{A}$, while $\operatorname{Blkdiag}\left(\mathbf{A}_{1}, \mathbf{A}_{2}, \cdots, \mathbf{A}_{N}\right)$ denotes a block diagonal matrix generated with the diagonal matrices $\mathbf{A}_{1}, \mathbf{A}_{2}, \cdots, \mathbf{A}_{N} . \mathbb{Z}$ and $\mathbb{Z}^{+}$refer to the set for non-negative and positive integers, respectively. $\bmod (a, b)$ denotes the modulo operation. $(\mathbf{A})^{\downarrow 2}$ denotes down sampling matrix $\mathbf{A}$ by taking its odd rows to form a new matrix.

\section{MS-SFMC SYSTEMS}

\section{A. Frequency domain}

Let us consider an MS communication system with system bandwidth $B$ offering total $K$ different services. The $k$-th service bandwidth is $B_{k}$ and the GB between the $k$-th and the $(k+1)$-th service is $\Delta B_{k}$. In addition, we assume $\Delta B_{0}$ and $\Delta B_{K}$, at edges of the frequency band, are GBs (or unoccupied bands) between the considered MS-SFMC system and other systems. The bandwidth allocation is shown in Fig. 2 (a). Without loss of generality, we assume the system bandwidth $B$ is normalized to unity, i.e., $B=\sum_{k=0}^{K} \Delta B_{k}+\sum_{k=1}^{K} B_{k}=1$.

For a system containing multiple types of services, it is natural to assume that each service supports one or more users, where each user can be granted arbitrary number of consecutive or non-consecutive physical resource blocks $(\mathrm{PRBs})^{1}$. Unlike single service multi-carrier system (e.g., LTE/LTEA) having a 3-tier frequency resource structure from system bandwidth, PRB and subcarrier, the MS-SFMC system has a 4-tier frequency resource structure: system bandwidth, service bandwidth, PRB to subcarrier. This not only complicates scheduling schemes on the MAC layer, but also affects the PHY layer algorithm design and subband filtering implementations. On which level does the filtering subband ${ }^{2}$ based will affect the SFMC system performance and implementation complexity. Fig. 2 (b-1), (b-2) and (b-3) shows subbands based on PRBs, user and service, respectively ${ }^{3}$.

There are pros and cons for different types of subband filtering schemes. PRB is the minimum scheduling granularity and the subband filtering based on one or more PRBs (Fig. 2 (b-1)) can gain the greatest design flexibility. In addition, relatively small subband bandwidth can effectively mitigate the channel dispersions and transceiver imperfections induced performance loss. On the other hand, this implementation also incurs the largest computational complexity among three of them due to the dense subband filtering operation. On the contrary, service based subband filtering method (Fig. 2 (b-3)) has the lowest computational complexity and the users (and PRBs) in one service share the same filter design parameters. However, different users in one service may suffer from different channel qualities, receiver sensitivities, etc. which can not be optimized by filter design independently. In the

\footnotetext{
${ }^{1}$ Note that here PRB is not necessarily the same as defined in LTE/LTE-A with 12 subcarriers. We reuse the terminology as minimum scheduling unit comprising of a number of consecutive subcarriers.

${ }^{2}$ To avoid confusion, we use subband as a frequency band filtered by a finite impulse response (FIR) filter, service bandwidth as the frequency band allocated to a type of service.

${ }^{3}$ Note that the system bandwidth can be filtered as a whole. However, this filtering method can not provide any flexibility, therefore, is not included in the discussion in this paper.
}

middle, user-based subband filtering as shown in Fig. 2 (b-2) is a compromised solution between PRB-based and servicebased methods. It enjoys the degree of freedom to adjust the filter design for specific user, meanwhile, the complexity is reduced comparing to the PRB-based implementation. For more generalized derivation, in this paper, we consider the PRB-based implementation.

\section{B. Time domain}

The symbol durations among services are different due to the different subcarrier spacings, which makes the (spectrum efficient) synchronization of the whole bandwidth unattractive and practically impossible. For example, in OFDM without considering the guard interval, two services with subcarrier spacing $\Delta f_{1}=2 \Delta f_{2}$ implies that the symbol duration has the relationship of $\Delta T_{2}=2 \Delta T_{1}$. Consequently, the symbols at service 1 can not synchronize with symbols in service 2 . However, we can take advantage of the fact that every 2 symbols in service 1 has the same duration as in service 2, and we call it a GS system, which is defined as

Definition 1: For the $k$-th service symbol duration being $\Delta T_{k}$, if there is an integral least common multiplier (LCM) duration $T_{L C M}$ for all services, satisfying

$$
\begin{aligned}
& T_{L C M}=N_{\text {sym }, 1} \Delta T_{1}=N_{\text {sym }, 2} \Delta T_{2}=\cdots=N_{\text {sym }, K} \Delta T_{K}, \\
& \text { with } \quad N_{\text {sym }, k} \in \mathbb{Z}^{+} \quad \text { for } \quad k=1,2, \cdots, K,
\end{aligned}
$$

we call the $K$ services are generally synchronized in one LCM symbol duration $T_{L C M}$.

One LCM symbol for a GS system is a closed space that does not affect and also is not affected by other symbols out of the considered LCM symbol. Fig 3 (a) is an example of the GS-MS system, where the services 1, 2 and 3 are GS with $N_{\text {sym }, 1}=1, N_{\text {sym }, 2}=2$ and $N_{\text {sym }, 3}=3$. The advantage of the GS system is that it simplifies system and algorithms design and performance analysis since only limited symbols need to be considered in a processing window and every LCM symbol has the same overall performance.

However, in a GS-SFMC system, the symbol duration plus overhead (such as filter tails and guard interval, etc.) should satisfy equation (1), which might reduce the degree of freedom for system design. In addition, when LCM is large for the MS system, taking one LCM symbol for joint processing leads to high processing complexity. In such cases, it is not necessary to keep a system generalized synchronized, and a non-synchronized MS system is given in Fig. 3 (b).

We will first focus on GS-MS system in Section III and then relax the constraints to a non-synchronized one in Section IV. In addition, the next derivations will be based on UFMC system [5], [6], [7], [17], which is one of the most widely used SFMC implementations in literature.

\section{GS-MS-UFMC SYSTEM MODEL, ISBI ANALYSIS AND CANCELATION ALGORITHMS}

\section{A. UFMC subband filtering}

Let us use $M_{k}$ as the number of subcarriers for the $k$-th service in an MS-UFMC system. Let us assume that the $k$-th 

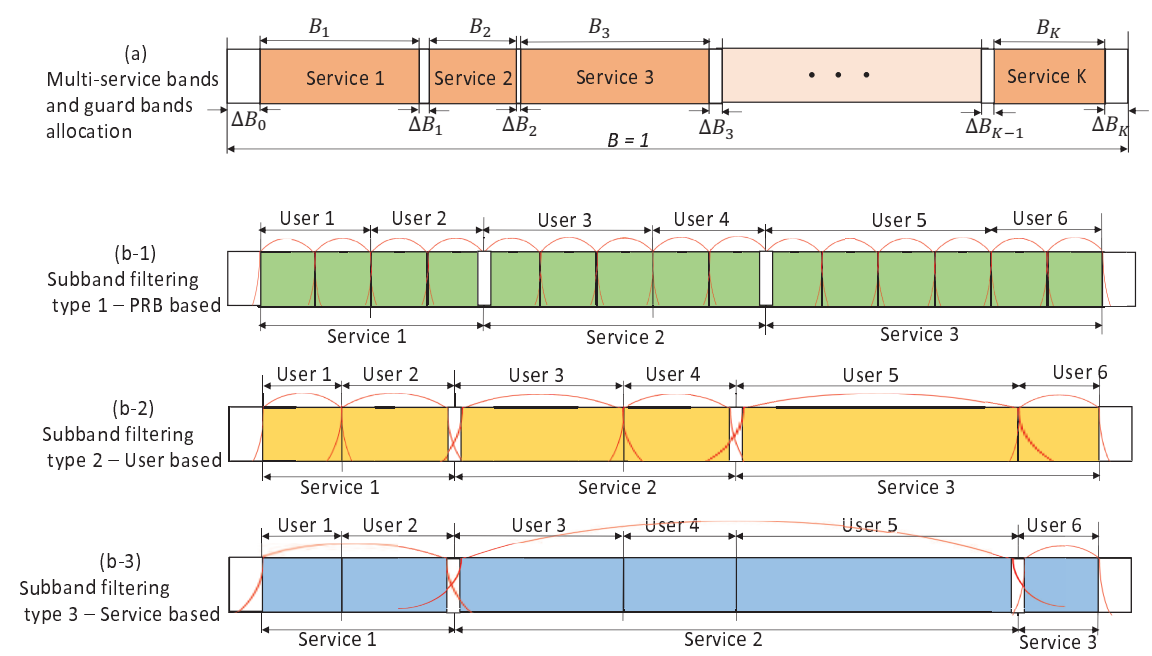

Fig. 2. (a): Service bands and guard bands allocation; (b): Three types of subband filtering methods. (note that the number of user, subband and service are for demonstration purpose only in this figure).

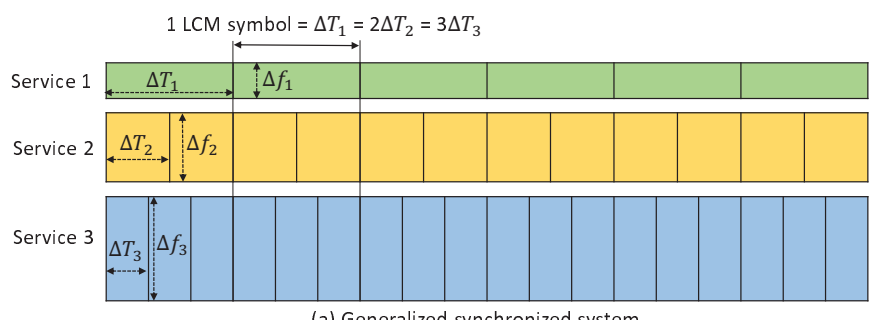

(a) Generalized synchronized system

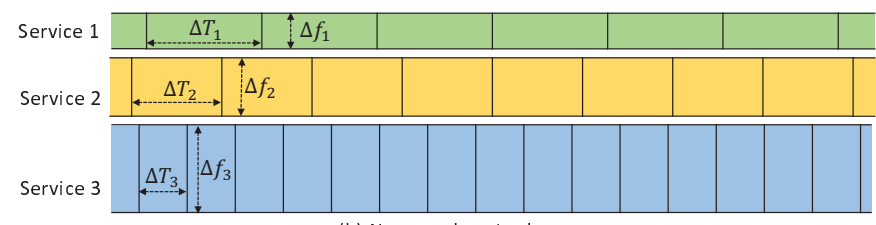

(b) Non-synchronized system

Fig. 3. Generalized synchronized and non-synchronized MS systems.

service contains $V_{k}$ subbands. In total, the system has $V=$ $\sum_{i=1}^{K} V_{k}$ subbands. In order to simplify the system model and derivations, we assume that the number of subcarriers in each subband in the $k$-th service is $Z_{k}$, thus we have $M_{k}=V_{k} Z_{k}$.

For a GS-MS-UFMC model which satisfies equation (1), the service with shorter symbol duration may overlap with different parts of the longer symbol (e.g., Fig. 3 (b)). To evaluate the complete system performance for all symbols, it is necessary to consider the performance over at least one LCM symbol duration, which can transmit $N_{\text {info }}=$ $\sum_{i=1}^{K} N_{s y m, k} M_{k}$ information symbols. Writing it in vector form:

$$
\begin{aligned}
& \mathbf{a}=[\underbrace{\mathbf{a}_{1,1} ; \mathbf{a}_{1,2} ; \cdots ; \mathbf{a}_{1, V_{1}}}_{=\mathbf{a}_{1}} ; \underbrace{\mathbf{a}_{2,1} ; \mathbf{a}_{2,2} ; \cdots ; \mathbf{a}_{2, V_{2}}}_{\mathbf{a}_{2}} ; \cdots ; \\
& \underbrace{\mathbf{a}_{K, 1} ; \mathbf{a}_{K, 2} ; \cdots ; \mathbf{a}_{K, V_{K}}}_{\mathbf{a}_{K}}],
\end{aligned}
$$

where $\mathbf{a}_{k}=\left[\mathbf{a}_{k, 1} ; \mathbf{a}_{k, 2} ; \cdots ; \mathbf{a}_{k, V_{k}}\right] \in \mathbb{C}^{N_{s y m, k} M_{k} \times 1}$ denotes the information symbol vector transmitted in the $k$-th service in one LCM symbol. $\mathbf{a}_{k, i}=\left[\mathbf{a}_{k, v, 1} ; \mathbf{a}_{k, v, 2} ; \cdots ; \mathbf{a}_{k, v, N_{s y m}, k}\right] \in$

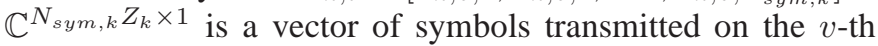
subband in the $k$-th service in one LCM symbol and $\mathbf{a}_{k, v, l}=$ $\left[a_{k, v, l}(0), a_{k, v, l}(1), \cdots, a_{k, v, l}\left(Z_{k}-1\right)\right]^{T} \in \mathbb{C}^{Z_{k} \times 1}$ denotes the information symbols transmitted in the $l$-th symbol of the LCM symbol and at the $v$-th subband of the $k$-th service. We assume that information symbols have zero mean and variance $\rho_{\text {sym }}^{2}$, i.e., $\mathcal{E}\left\{a_{k, v, l}(i)\right\}=0$ and $\mathcal{E}\left\{\left|a_{k, v, l}(i)\right|^{2}\right\}=\rho_{\text {sym }}^{2}$.

Let us assume that the filter for the $v$-th subband of the $k$-th service is $\mathbf{f}_{k, v}=\left[f_{k, v}(0), f_{k, v}(1), \cdots, f_{k, v}\left(L_{F, k}-1\right)\right]$, where $L_{F, k}$ is the filter length for the $k$-th service. We assume that the filter length for all subbands in one service bandwidth is the same to simplify the system model and derivations. Without loss of generality, we assume that the power of $\mathbf{f}_{k, v}$ is normalized to unity i.e. $\sum_{i=0}^{L_{F, k}-1}\left|f_{k, v}(i)\right|^{2}=1$.

Note that each subband processing in multi-service UFMC system is exactly the same as the single service one [5], [6], [7], [17], but with different system parameters among services, where the discrete Fourier transform (DFT) size for the $k$-th service is $N_{k}=M_{k} / B_{k} \in \mathbb{Z}^{+}$with $B_{k}$ being the bandwidth for the $k$-th service. Let us assume $L_{k} \geq N_{k}+L_{F, k}-1$ is the UFMC symbol length (in samples) of the $v$-th subband of the $k$-th service. We define $\mathbf{A}_{k, v} \in \mathbb{C}^{L_{k} \times N_{k}}$ as a Toeplitz matrix with its first column and first row being $\tilde{\mathbf{f}}_{k, v}=$ $\left[\mathbf{f}_{k, v}, \mathbf{0}_{1 \times\left(L_{k}-L_{F, k}\right)}\right]^{T}$ and $\left[f_{k, v}(0), \mathbf{0}_{1 \times\left(N_{k}-1\right)}\right]$, respectively. Following the same processing as single service UFMC [5], [22], we can write the inverse DFT (IDFT) transformed and subband filtered signal at the $i$-th symbol and $v$-th subband of the $k$-th service as:

$\mathbf{q}_{k, v, i}=\frac{1}{\rho_{k, v}} \mathbf{f}_{k, v} *\left(\mathbf{D}_{k, v} \mathbf{a}_{k, v, i}\right)=\frac{1}{\rho_{k, v}} \mathbf{A}_{k, v} \mathbf{D}_{k, v} \mathbf{a}_{k, v, i}$,

where $\mathbf{D}_{k, v} \in \mathbb{C}^{N_{k} \times Z_{k}}$ is a submatrix of the $N_{k}$-point normalized and frequency shifted IDFT matrix. The element in $l$-th row and $n$-th column of $\mathbf{D}_{k, v}$ is $d_{l, n}=\frac{1}{\sqrt{N_{k}}} e^{j 2 \pi l\left(n+\eta_{k, v}\right) / N_{k}}$, where $\eta_{k, v}$ is the frequency shift for the $v$-th subband in the 
$k$-th service and given as:

$$
\eta_{k, v}=N_{k}\left(\sum_{m=1}^{k-1} B_{m}+\sum_{m=0}^{k-1} \Delta B_{m}\right)+\left(V_{k}-1\right) Z_{k, m} .
$$

$\rho_{k, v}=\sqrt{\frac{1}{Z_{k}} \operatorname{trace}\left(\mathbf{D}_{k, v}^{H} \mathbf{A}_{k, v}^{H} \mathbf{A}_{k, v} \mathbf{D}_{k, v}\right)}$ is the transmission power normalization factor. Due to the filter tail and zero padding, $\mathbf{q}_{k, v, i} \in \mathbb{C}^{L_{k} \times 1}$ is $L_{k}-N_{k}$ samples longer than the IDFT operated signal $\mathbf{D}_{k, v} \mathbf{a}_{k, v, i}$, which is the overhead of the service in time domain.

Note that equation (3) is a unified expression for two scenarios. When the symbol duration $L_{k}=N_{k}+L_{F, k}-1$, it implies that the total overhead is generated by the filter tails and no guard interval is inserted between symbols. On the other hand, when $L_{k}>N_{k}+L_{F, k}-1$, we define $L_{Z P, k}=L_{k}-\left(N_{k}+L_{F, k}-1\right)$ as the guard interval between symbols to combat the multipath channel. Specifically, we pad $L_{Z P, k}$ zeros at the end of the subband filtered signal. According to [22], when ZP length is larger than or equal to the channel length minus one, it is an ISI free system in one service band.

\section{B. GS-MS-UFMC system model}

Different from the single service UFMC, we have to consider a whole LCM symbol to model a complete GS-MSUFMC system model. For the GS system which satisfies (1), the following equation also holds true:

$$
L_{L C M}=N_{s y m, 1} L_{1}=\cdots=N_{s y m, K} L_{K} .
$$

(5) is different from (1) since it expresses the GS conditions using number of samples instead of using symbol duration in seconds. $L_{L C M}$ refers to the total number of samples in one LCM symbol. Then the total LCM samples in the $v$-th subband of the $k$-th service can be expressed as:

$$
\mathbf{q}_{k, v}=\left[\mathbf{q}_{k, v, 1} ; \mathbf{q}_{k, v, 2} ; \cdots ; \mathbf{q}_{k, v, N_{s y m}, k}\right]=\frac{1}{\rho_{k, v}} \mathbf{G}_{k, v} \mathbf{a}_{k, v}
$$

where

$$
\mathbf{G}_{k, v}=\operatorname{Blkdiag}\left(\mathbf{A}_{k, v} \mathbf{D}_{k, v}, N_{s y m, k}\right) \in \mathbb{C}^{L_{L C M} \times N_{s y m, k} Z_{k}}
$$

By considering all subbands and all services in the system, the signal before transmission over the channel can be written as:

$$
\mathbf{p}=\sum_{k=1}^{K} \sum_{v=1}^{V_{k}} \mathbf{q}_{k, v} \in \mathbb{C}^{L_{L C M} \times 1}
$$

Let us assume the channel between the transmitter and the $m$-th user is $\mathbf{h}_{m}=\left[h_{m}(0), h_{m}(1), \cdots, h_{m}\left(L_{C H, m}-1\right)\right]$, where $L_{C H, m}$ is the length of the channel in samples. Using equation (8), the received signal at the $m$-th user can be written as $\mathbf{y}_{m}=\mathbf{h}_{m} * \mathbf{p}+\mathbf{w}_{m}$, where the noise vector $\mathbf{w}_{m}=\left[w_{m}(0), w_{m}(1), \cdots, w_{m}\left(L_{L C M}-1\right)\right]^{T}$ with $w_{m}(l) \sim$ $\mathcal{C N}\left(0, \sigma^{2}\right)$.

At the $m$-th receiver that belongs to the $k$-th service, $\mathbf{y}_{m}$ is split into $N_{\text {sym, } k}$ non-overlapping UFMC symbols each being a length $L_{k}$ (i.e., symbol duration of the $k$-th service) vector for receiver baseband processing. Let us define $\mathbf{y}_{m, i}, \mathbf{q}_{n, l, i}$ and $\mathbf{w}_{m, i}$ as the $\left[(i-1) L_{k}+1\right]$-th to $i L_{k}$-th element of $\mathbf{y}_{m}, \overline{\mathbf{q}}_{n, l}$ and $\mathbf{w}_{m}$, respectively. Then the $i$-th received UFMC symbol of the $m$-th user allocated to the $v$-th subband of the $k$-th service can be written as:

$$
\begin{aligned}
& \mathbf{y}_{m, i}=\mathbf{B}_{m} \overline{\mathbf{q}}_{k, v, i}+\mathbf{B}_{m} \sum_{k=1}^{K} \sum_{l=1, l \neq v}^{V_{k}} \overline{\mathbf{q}}_{k, l, i}+\mathbf{B}_{m} \sum_{n=1, n \neq k}^{K} \sum_{n=1}^{V_{n}} \overline{\mathbf{q}}_{n, l, i}+ \\
& \underset{\mathbf{B}_{m}}{\sum_{n=1, n \neq k}^{K}} \sum_{l=1}^{V_{n}} \overline{\mathbf{q}}_{n, l, i-1}+\mathbf{y}_{m, i, I S I}+\mathbf{w}_{m, i}
\end{aligned}
$$

where $\mathbf{B}_{m} \in \mathbb{C}^{L_{k} \times L_{k}}$ is the equivalent Toeplitz channel convolution matrix of $\mathbf{h}_{m}$, with first column $\left[\mathbf{h}_{m}, \mathbf{0}_{\left(L_{k}-L_{C H, m}\right) \times 1}\right]^{T}$ and first row $\left[h_{m}(0), \mathbf{0}_{\left(L_{k}-1\right) \times 1}\right]$. $\Delta \mathbf{B}_{m}$ is an upper-triangle matrix with the $l$-th row and $l+\left(L_{m}-L_{C H, m}\right)+m$-th column being $h_{m}(l+m)$ for $l, m=1,2 \cdots, L_{C H, m}-1$, while other elements are zeros. The first term in equation (9) is the desired signal and the last term represents the noise. The second term is the ICI that is defined as the interference generated from other subcarriers in the same UFMC symbol of the $k$-th service. The 5 -th term in equation (9) is the ISI defined as the interference generated from the last symbol of the $k$-th service, while the third and 4-th terms represent ISBI that is the interference from other services to the $k$-th service. When $L_{Z P, k} \geq L_{C H, m}-1$, $\mathbf{y}_{m, i, I S I}=\mathbf{0}$ [22]. In this section, we assume that ZP length is sufficient and $\mathbf{y}_{m, i, I S I}=\mathbf{0}$ in order to focus on the ISBI performance analysis. The generalized case with arbitrary length $\mathrm{ZP}$ will be derived in the next section.

According to the UFMC receiver processing [5], $\mathbf{y}_{m, i}$ is zero-padded to yield $\tilde{\mathbf{y}}_{m, i}=\left[\mathbf{y}_{m, i} ; \mathbf{0}_{\left(2 N_{k}-L_{k}\right) \times 1}\right]$. Then $2 N_{k}$-point DFT is performed and signal is down-sampled by a factor of 2 . Then taking the first $Z_{k}$ rows, we have $\mathbf{c}_{m, i}=\left(\tilde{\mathbf{D}}_{k, v}^{H} \tilde{\mathbf{y}}_{m, i}\right)^{\downarrow 2}$. As proved in Appendix A, $\mathbf{c}_{m, i}$ can be expressed as

$$
\mathbf{c}_{m, i}=\frac{1}{\rho_{k, v}} \mathbf{H}_{m, v} \mathbf{F}_{k, v} \mathbf{a}_{k, v, i}+\mathbf{y}_{I S B I, m, i}+\tilde{\mathbf{w}}_{m, i},
$$

where $\tilde{\mathbf{w}}_{m, i}$ is the DFT operated noise vector with $l$-th element $\sum_{n=0}^{L_{k}-1} e^{-j 2 \pi\left(l+\eta_{k, v}\right) n / N_{k}} w_{m, i}(n) . \mathbf{H}_{m, v}$ and $\mathbf{F}_{k, v}$ are channel and filter frequency responses, which are $Z_{k}$ dimension diagonal matrices with the $l$-th diagonal element being $H_{m, v}(l)=\sum_{n=0}^{L_{C H, m}-1} h_{m, v}(n) e^{-j 2 \pi n\left(l+\eta_{k, v}\right) / N_{k}}$ and $F_{k, v}(l)=\sum_{n=0}^{L_{F, k}-1} f_{k, v}(n) e^{-j 2 \pi n\left(l+\eta_{k, v}\right) / N_{k}}$, respectively. $\mathbf{y}_{I S B I, m, i}$ is ISBI contributed by the third and fourth terms in (9) and can be written as:

$$
\begin{aligned}
& \mathbf{y}_{I S B I, m, i}=\sum_{n=1, n \neq k}^{K} \sum_{l=1}^{V_{n}} \frac{1}{\rho_{n, l}}\left(\mathbf{H}_{m, v} \hat{\mathbf{D}}_{k, v}^{H} \tilde{\mathbf{G}}_{n, l, i} \mathbf{a}_{n, l}\right. \\
& \left.+\hat{\mathbf{D}}_{k, v}^{H} \mathbf{\Delta} \mathbf{B}_{m} \mathbf{G}_{n, l, i-1} \mathbf{a}_{n, l}\right),
\end{aligned}
$$

$\hat{\mathbf{D}}_{k, v}^{H}=\left(\tilde{\mathbf{D}}_{k, v}^{H}\right)^{\downarrow 2}$, and $\tilde{\mathbf{D}}_{k, v}^{H} \in \mathbb{C}^{2 Z_{k} \times 2 N_{k}}$ contains the first $2 Z_{k}$ rows of frequency shifted $2 N_{k}$ dimension DFT matrix $\mathbf{D}_{2 N_{k}}^{H}$ and its $l$-th row and $n$-th column is $e^{-j 2 \pi\left(l+2 \eta_{k, v}\right) n /\left(2 N_{k}\right)} \cdot \tilde{\mathbf{G}}_{n, l, i}=\left[\mathbf{G}_{n, l, i} ; \mathbf{0}_{\left(2 N_{k}-L_{k}\right) \times N_{s y m, n} Z_{n}}\right]$ and $\mathbf{G}_{n, l, i}$ is a sub-matrix of $\mathbf{G}_{n, l}$ obtained by taking its $\left[(i-1) L_{k}+1\right]$-th to $i L_{k}$-th rows. 
The first term of (10) is the desired signal only and one-tap channel equalization can be performed since $\mathbf{H}_{m, v} \mathbf{F}_{k, v}$ is a diagonal matrix. The ISBI $\mathbf{y}_{I S B I, m, i}$ defined in (11) comprises of two terms, the first one $\sum_{n=1, n \neq k}^{K} \sum_{l=1}^{V_{n}} \frac{1}{\rho_{n, l}} \mathbf{H}_{m, v} \hat{\mathbf{D}}_{k, v}^{H} \tilde{\mathbf{G}}_{n, l, i} \mathbf{a}_{n, l}$ has also been channelized and therefore can be used for low complexity ISBI cancelation algorithm in the next subsection. However, the second one, $\frac{1}{\rho_{n, l}} \sum_{n=1, n \neq k}^{K} \sum_{l=1}^{V_{n}} \hat{\mathbf{D}}_{k, v}^{H} \mathbf{\Delta} \mathbf{B}_{m} \mathbf{G}_{n, l, i-1} \mathbf{a}_{n, l}$ is negligible with moderate channel delay spread since the elements of $\mathbf{\Delta} \mathbf{B}_{k, v}$ consist of small component of the channel response. Note that the index $i-1$ in $\mathbf{G}_{n, l, i-1}$ refers to the interference from the last symbol and when $i=1, \mathbf{G}_{n, l, 0}$ denotes the interference from the last LCM symbol.

\section{ISBI analysis}

Equation (10) gives a complete signal model for a GSMS-UFMC system in the absence of transceiver errors and sufficient guard interval between symbols. However, this expression does not show how do the differences in subcarrier spacing and GB between adjacent services affect the ISBI level. In addition, due to the complex expression, it is also not straightforward to see how the subband filtering attenuates the ISBI from the adjacent service band and outperforms OFDM system, which is identified as one of the most promising characteristics of this new waveform. Therefore, we focus on ISBI analysis and the properties.

Note that non-adjacent service bands do not generate significant ISBI and affect the performance. Therefore, we can consider two types of services adjacent to each other in the frequency band (e.g., subband 4 and 5 in Fig. 2 (b-1) ) and each one is allocated 1 subband only to simplify the analysis. In addition, without loss of generality, we assume $\Delta B_{0}=0$. Then we have the following proposition 1:

Proposition 1: Consider a GS-MS-UFMC system with two services and subcarrier spacing $\Delta f_{1}=M_{s c} \Delta f_{2}$ for $M_{s c} \in \mathbb{Z}^{+}$and the guard band $\Delta B_{1}$. In addition, the $Z P$ length for both services satisfies $L_{Z P, 1} \geq L_{C H, 2}-1$ and $L_{Z P, 2} \geq L_{C H, 2}-1$. Then the power of ISBI at the l-th subcarrier of service 2 can be written as:

$$
P_{2, I S B I}(l)=\frac{\sigma_{\text {sym }}^{2}}{\rho_{1,1}^{2}}\left|H_{2,1}(l)\right|^{2}\left|\tilde{F}_{1}(l)\right|^{2} J_{1}(l),
$$

where

$$
\tilde{F}_{1}(l)=\sum_{n=0}^{L_{F, 1}-1} e^{-j 2 \pi\left(l+\eta_{1,1}\right) n / N_{2}} f_{1,1}(n)
$$

is frequency shifted filter response of subband 1 in frequency domain.

$$
J_{1}(l)=\sum_{k=0}^{M_{s c} Z_{1}-1}\left|\sum_{n=0}^{N_{1}-1} e^{-j 2 \pi n\left[\frac{l}{N_{1}}-\frac{k+\left(B_{1}+\Delta B_{1}\right) N_{1}}{M_{s c} N_{1}}\right]}\right|^{2}
$$

is the spectrum leakage factor from the subband 1 to subband 2 and it is constant in both UFMC and OFDM systems.

Proof: See Appendix B.

Equation (12) is written as a multiplication of four terms with the first and second being the transmitter power and channel gain, respectively. The third term $\left|\tilde{F}_{1}(l)\right|^{2}$ is attributed to the waveform (i.e., filter response), which is not related to the MS system parameters (e.g., subcarrier spacing differences). The fourth term $J_{1}(l)$ is due to the the subcarrier spacing difference between the two adjacent services, which is not coupled with the filter response. In a single-service system, $M_{s c}=1$, resulting in $J_{1}(l)=0$ and $P_{2, I S B I}(l)=0$, in such a case, the system is orthogonal.

Note that for the filter frequency response in subband 1, $F_{1,1}(l)=\sum_{n=0}^{L_{F, 1}} e^{-j 2 \pi l n / N_{2}} f_{1,1}(n)$, its pass band is indexed $0 \leq l \leq M_{1}-1$ and $l>M_{1}-1$ is the stop band of the filter. However, $\tilde{F}_{1}(l)$ is a frequency shifted version of filter response $F_{1,1}(l)$ and when $0 \leq l \leq M_{1}-1, \tilde{F}_{1}(l)$ is the filter response from its stop band, resulting in ISBI mitigation to subband 2.

$\tilde{F}_{1}(l)$ attenuates the ISBI from subband 1. However, for the subcarriers at the edge of the subband 2 , the first subcarrier has the filter response $\tilde{F}_{1}(0)=$ $\sum_{n=0}^{L_{F, 1}-1} e^{-j 2 \pi\left(n M_{1} / N_{1}+n \Delta B_{1} / M_{s c}\right)} f_{1,1}(n)$, i.e., it is frequency shifted $\Delta B_{1} / M_{s c}$ from its last subcarrier of the pass band. With small $\Delta B_{1}$, ISBI might be significant. Large $\Delta B_{1}$ will push $\tilde{F}_{1}(l)$ away from the pass band of $F_{1}(l)$ which results in better ISBI mitigation. This indicates that larger guard band may be required for reliable transmissions.

Note that Proposition 1 is for general case of UFMC system. By taking the filter $\mathbf{f}=1$ and $\tilde{F}_{1}(l)=1$, it is equivalent to MS OFDM systems: $P_{2, I S B I}^{o f d m}(l)=\frac{\sigma_{s y m}^{2}}{\rho_{1,1}^{2}}\left|H_{2,1}(l)\right|^{2} J_{1}(l)$. Comparing it with (12) for UFMC system, the interference in UFMC system is attenuated by the filter response $\left|\tilde{F}_{1}(l)\right|^{2}$ while OFDM system does not have this advantage.

Proposition 2: Consider the same GS-MS-UFMC system in Proposition 1, and the guard band $\Delta B_{1}=M_{g b} \Delta f_{2}$ with $M_{g b} \in \mathbb{Z}$ and $M_{g b} \geq 0$, we have

$$
P_{2, I S B I}(l)=0 ; \quad \text { for } \quad l=\bmod \left(M_{g b}, M_{s c}\right)+m M_{s c}
$$

i.e., the $\left[\left(\bmod \left(M_{g b}, M_{s c}\right)+m M_{s c}+1\right]\right.$-th subcarriers in subband 2 are not affected by ISBI for $m \in \mathbb{Z}^{+}$

Proof: See Appendix B.

Proposition 2 implies that every $M_{s c}$ subcarriers from the $\left[\left(\bmod \left(M_{g b}, M_{s c}\right)+1\right)\right]$-th subcarrier, are ISBI free. This fact can be leveraged to design a symbol mapping scheme to maximize the performance of the system. For example, the reference signals can be scattered at those subcarriers to increase the channel estimation accuracy. Again, Proposition 2 is a general case and also applicable to MS OFDM system, i.e., $P_{2, I S B I}^{o f d m}(l)=0$, for $l=\bmod \left(M_{g b}, M_{s c}\right)+m M_{s c}$.

Propositions 1 and 2 describe the ISBI from the subband with larger subcarrier spacing to the smaller one. Now let us move in the opposite direction:

Proposition 3: Consider the same GS-MS-UFMC system in Proposition 1 with the assumption $L_{1}>>L_{F, 2}$. Then the ISBI at the l-th subcarrier and the $i$-th symbol of service 1 can be written as:

$$
P_{1, I S B I}(l, i) \approx \frac{\sigma_{\text {sym }}^{2}}{\rho_{2,1}^{2}}\left|H_{1,1}(l)\right|^{2}\left|\tilde{F}_{2}(l)\right|^{2} J_{2}(l)
$$


where

$$
\tilde{F}_{2}(l)=\sum_{n=0}^{L_{F, 2}-1} e^{-j 2 \pi n l / N_{1}}
$$

is the frequency domain filter response.

$$
J_{2}(l)=\sum_{k=0}^{Z_{2}-1}\left|\sum_{n=0}^{L_{1}-1} e^{-j 2 \pi \frac{M_{s c} l n-\left(n+(i-1) L_{1}\right)\left(k+\eta_{2,1}\right)}{M_{s c N_{1}}}}\right|^{2}
$$

Proof: See Appendix C.

Similar to the Proposition $1, P_{1, I S B I}(l, i)$ is written as a product of 4 terms including the filter response $\left|\tilde{F}_{2}(l)\right|^{2}$ and the subcarrier spacing differences induced factor $J_{2}(l)$. Unlike Proposition 1, $P_{1, I S B I}(i, l)$ in Proposition 3 is controlled by two parameters: index of subcarrier and symbol, the later one is due to the symbol duration of service 2, which is $M$ times longer compared to service 1 . Consequently, different symbols in service 1 overlap with different parts of symbol of service 2 , resulting in different ISBI.

Again, we can see that the filter response in frequency domain (i.e., $\left|\tilde{F}_{2}(l)\right|^{2}$ ) attenuates the ISBI from subband 2. Larger $\Delta B_{1}$ results in better ISBI mitigation. In addition, by taking $\left|\tilde{F}_{2}(l)\right|^{2}=1$, (16) will be degraded to the OFDM system.

\section{Proposed ISBI cancelation algorithms}

According to Proposition 1, 2, 3, some subcarriers at the edge of service bands may suffer from serious interference, leading to performance loss. In this subsection, we propose baseband processing algorithms at the transmitter to eliminate the ISBI. To simplify the derivations and expressions, we will consider an MS system with two services and each service contains only one subband. However, it is trivial to extend the algorithms to a general case. Note that we will relax some assumptions in Proposition 1, 2 and 3, specifically, we consider arbitrary $M_{s c}$ and $M_{g b}$.

Even within the two adjacent subbands, the ISBI may only affect limited number of subcarriers. Therefore, to further reduce the complexity and improve the flexibility of algorithm design, let us assume that the last $N_{c a n, 1}$ and first $N_{c a n, 2}$ subcarriers of the first service and the second service are contaminated by severe ISBI (and also generate the highest interference) and are considered in the cancelation algorithms.

It is natural to assume the two subbands belong to two different users. According to equation (11), we can combine the received signal of user 1 and 2 together as follows:

$$
\mathbf{c}_{c}=\mathbf{H}_{c} \mathbf{E} \mathbf{a}_{c}+\mathbf{b}_{c}
$$

where $\mathbf{H}_{c}=\operatorname{Blkdiag}\left\{\overline{\mathbf{H}}_{1, \text { all }}, \overline{\mathbf{H}}_{2, \text { all }}\right\}$ and $\overline{\mathbf{H}}_{1, \text { all }}=$ $\operatorname{Blkdiag}\left(\overline{\mathbf{H}}_{1,1}, N_{\text {sym }, 1}\right)$ and $\overline{\mathbf{H}}_{2, \text { all }}=\operatorname{Blkdiag}\left(\overline{\mathbf{H}}_{2,1}, N_{\text {sym }, 2}\right)$ with $\overline{\mathbf{H}}_{1,1}$ being sub-matrix of channel matrix $\mathbf{H}_{1,1}$ obtained by taking the last $N_{c a n, 1}$ columns and rows. While $\overline{\mathbf{H}}_{2,1}$ is the sub-matrix of $\mathbf{H}_{2,1}$ generated from the first $N_{c a n, 2}$ columns and rows. $\mathbf{a}_{c}$ is the considered information symbols vector for ISBI cancelation and can be expressed as

$$
\mathbf{a}_{c}=\left[\overline{\mathbf{a}}_{1,1,1}, \cdots, \overline{\mathbf{a}}_{1,1, N_{s y m}, 1}, \overline{\mathbf{a}}_{2,1,1}, \cdots, \overline{\mathbf{a}}_{2,1, N_{s y m, 2}}\right]^{T}
$$

and $\overline{\mathbf{a}}_{1,1, i}=\left[a_{1,1, i}\left(Z_{1}-N_{c a n, 1}\right), a_{1,1, i}\left(Z_{1}-N_{c a n, 1}+\right.\right.$ 1), $\left.\cdots, a_{1,1, i}\left(Z_{1}-1\right)\right]$ comprises of the last $N_{c a n, 1}$ symbols of $\mathbf{a}_{1,1, i}$ for ISBI cancelation. Obviously, $N_{c a n, 1}=$ $Z_{1}$ implies a special case that all of the subcarriers are considered in the cancelation algorithm. Similarly, $\overline{\mathbf{a}}_{2,1, i}=\left[a_{2,1, i}(0), a_{2,1, i}(1), \cdots, a_{2,1, i}\left(N_{c a n, 2}-1\right)\right]$ refers to the first $N_{c a n, 2}$ symbols of $\mathbf{a}_{2,1, i}$ for ISBI cancelation. $\mathbf{b}_{c}=\tilde{\mathbf{w}}_{c}+\tilde{\mathbf{b}}_{c}$ and $\tilde{\mathbf{b}}_{c}=\left[\tilde{\mathbf{B}}_{c, 1} \mathbf{a}_{2,1} ; \tilde{\mathbf{B}}_{c, 2} \mathbf{a}_{1,1}\right]$, where $\tilde{\mathbf{B}}_{c, 1}=\frac{1}{\rho_{2,1}} \operatorname{Blkdiag}\left(\overline{\mathbf{D}}_{1,1}^{H} \mathbf{\Delta} \mathbf{B}_{1}\left[\mathbf{G}_{2,1,1}, \cdots, \mathbf{G}_{2,1, N_{s y m}, 1}\right]\right)$ with $\overline{\mathbf{D}}_{1,1}^{H}$ being the last $N_{c a n, 1}$ rows of $\tilde{\mathbf{D}}_{1,1}^{H}$ and $\tilde{\mathbf{B}}_{c, 2}=$ $\frac{1}{\rho_{1,1}} \operatorname{Blkdiag}\left(\overline{\mathbf{D}}_{2,1}^{H} \mathbf{\Delta} \mathbf{B}_{2}\left[\mathbf{G}_{1,1,1}, \cdots, \mathbf{G}_{1,1, N_{s y m, 2}}\right]\right)$ with $\overline{\mathbf{D}}_{2,1}^{H}$ being the first $N_{c a n, 2}$ rows of $\tilde{\mathbf{D}}_{2,1}^{H}$. In addition,

$$
\mathbf{E}=\left(\begin{array}{ll}
\mathbf{E}_{1,1} & \mathbf{E}_{1,2} \\
\mathbf{E}_{2,1} & \mathbf{E}_{2,2}
\end{array}\right)
$$

with

$$
\begin{aligned}
& \mathbf{E}_{1,1}=\frac{1}{\rho_{1,1}} \operatorname{Blkdiag}\left(\overline{\mathbf{F}}_{1,1}, N_{\text {sym }, 1}\right) \\
& \mathbf{E}_{2,2}=\frac{1}{\rho_{2,1}} \operatorname{Blkdiag}\left(\overline{\mathbf{F}}_{2,1}, N_{\text {sym }, 2}\right)
\end{aligned}
$$

$\mathbf{E}_{1,2}=\frac{1}{\rho_{2,1}} \operatorname{Blkdiag}\left(\hat{\mathbf{D}}_{1,1,2 N_{1}} \cdot\left[\overline{\mathbf{G}}_{2,1,1}, \cdots, \overline{\mathbf{G}}_{2,1, N_{s y m, 2}}\right]\right)$

$$
\mathbf{E}_{2,1}=\frac{1}{\rho_{1,1}} \operatorname{Blkdiag}\left(\hat{\mathbf{D}}_{2,1,2 N_{2}} \cdot\left[\overline{\mathbf{G}}_{1,1,1}, \cdots, \overline{\mathbf{G}}_{1,1, N_{s y m}, 1}\right]\right)
$$

where $\overline{\mathbf{F}}_{1,1}$ is the sub-matrix of filter response $\mathbf{F}_{1,1}$ generated by taking the last $N_{c a n, 1}$ columns and rows. $\overline{\mathbf{F}}_{2,1}$ is the sub-matrix of $\mathbf{F}_{2,1}$ taking its first $N_{c a n, 2}$ columns and rows. $\overline{\mathbf{G}}_{1,1, i}$ consists of the $\left[(i-1) L_{2}+1\right]$-th to the $i L_{2}$ th rows of $\overline{\mathbf{G}}_{1,1}=\operatorname{Blkdiag}\left(\mathbf{A}_{1,1} \overline{\mathbf{D}}_{1,1}, N_{\text {sym }, 1}\right)$ with $\overline{\mathbf{D}}_{1,1}$ obtained by taking the last $N_{\text {can }, 1}$ columns of $\mathbf{D}_{1,1}$. Similarly, $\overline{\mathbf{G}}_{2,1, i}$ consists of $\left[(i-1) L_{1}+1\right]$-th to the $i L_{1}$-th rows of $\overline{\overline{\mathbf{G}}}_{2,1}=\operatorname{Blkdiag}\left(\mathbf{A}_{2,1} \overline{\mathbf{D}}_{2,1}, N_{\text {sym }, 2}\right)$ with $\overline{\mathbf{D}}_{2,1}$ comprising of the first $N_{c a n, 2}$ columns of $\mathbf{D}_{2,1}$.

The matrix $\mathbf{E} \in \mathbb{C}^{N_{d} \times N_{d}}$ and $N_{d}=N_{d 1}+N_{d 2}$, where $N_{d 1}=N_{\text {can }, 1} N_{\text {sym }, 1}$ and $N_{d 2}=N_{\text {can }, 2} N_{\text {sym }, 2} . \mathbf{E}_{1,1} \in$ $\mathbb{C}^{N_{d 1} \times N_{d 1}}$ and $\mathbf{E}_{2,2} \in \mathbb{C}^{N_{d 2} \times N_{d 2}}$ are diagonal matrices, which implies that the subcarriers belonging to different services do not cause interference to each other.

With the constructed interference matrix $\mathbf{E}$, we can design a precoding algorithm to eliminate the ISBI for the considered subcarriers by solving the following optimization problem:

$\min _{\mathbf{P}}\left\|\mathbf{H}_{c} \mathbf{E P} \mathbf{a}_{c}+\mathbf{b}_{c}-\mathbf{a}_{c}\right\|^{2} ; \quad$ s.t. $\quad \operatorname{trace}\left(\mathbf{P} \mathbf{P}^{H}\right)=N_{d}$

where $\mathbf{P}$ is the precoding matrix to cancel ISBI. Based on the most widely used linear processing algorithms design criteria: ZF and MMSE, we have the following precoding algorithms:

$\mathbf{P}=\left\{\begin{array}{lc}\rho_{z f} \mathbf{E}^{-1} & \text { ZF } \\ \rho_{m m s e}\left(\mathbf{E}^{H} \mathbf{H}_{c}^{H} \mathbf{H}_{c} \mathbf{E}+\mathbf{U}_{c}\right)^{-1} \mathbf{E}^{H} \mathbf{H}_{c}^{H} & \text { MMSE }\end{array}\right.$

where

$$
\rho_{z f}=\sqrt{\frac{N_{d}}{\operatorname{trace}\left[\mathcal{E}\left(\left(\mathbf{E}^{-1}\right)^{H} \mathbf{E}^{-1}\right)\right]}}
$$


and

$\rho_{m m s e}=\sqrt{\frac{N_{d}}{\operatorname{trace}\left[\mathcal{E}\left(\mathbf{H}_{c} \mathbf{E}\left(\mathbf{E}^{H} \mathbf{H}_{c}^{H} \mathbf{H}_{c} \mathbf{E}+\sigma_{c}^{2}\right)^{-2} \mathbf{E}^{H} \mathbf{H}_{c}^{H}\right)\right]}}$

are power normalization factors for ZF and MMSE based algorithms, respectively. $\mathbf{U}_{c}=\rho_{\text {sym }}^{2} \operatorname{diag}\left(\tilde{\mathbf{U}}_{c} \tilde{\mathbf{U}}_{c}^{H}\right)+\sigma^{2} \mathbf{I}_{N_{d}}$ is the summation of power of interference and noise and $\tilde{\mathbf{U}}=\left[\tilde{\mathbf{B}}_{c, 1} ; \tilde{\mathbf{B}}_{c, 2}\right]$.

For the proposed ISBI analysis and cancelation algorithm, we have the following remarks:

- The signal model and ISBI analysis proposed in Section III-B and III-C are applicable to both uplink and downlink transmissions. However, the proposed ISBI cancelation algorithm in equation (27) is based on the downlink transmission. In such case, the transmitter has all of the required information to pre-cancel the ISBI. For the uplink transmission, since the information transmitted by different services may belong to different users, precoding is impractical. However, the receiver obtains all of the necessary information and the joint detection algorithms could be applied.

- MMSE-based precoding algorithm depends on instantaneous channel information, which could be obtained by the channel feedback or exploiting the channel reciprocity property of the time division duplex (TDD) system. The noise variance could be estimated or fedback by the receiver. The ZF-based precoding matrix depends on the filter design (i.e., E) and other fixed system related parameters, thus, can be calculated offline in advance.

- The ISBI cancelation algorithm at the transmitter may affect the system performance in terms of the peak-toaverage-ratio (PAPR) and the OoB emission. However, only several subcarriers on the the edge subbands will generate and be affected by ISBI significantly. In addition, the non-trivial elements of the precoding matrix $\mathbf{P}$ are located close to the main diagonal. Consequently, only few subcarriers' power is changed, resulting in negligible performance loss in terms of PAPR and OoB emission.

- Note that pre-compensating the signal power among the subcarriers in one subband may improve the overall performance of a UFMC system in the single-service system, since it can evenly allocate the power among the subcarriers. However, this concept does not conflict with the proposed ISBI analysis and the cancelation algorithms in multi-service system. In fact, power precompensation can work under the proposed multi-service system framework as a performance enhancer.

- Since the optimization problem in (26) minimizes the overall estimation error and considers all symbols in the cancelation bandwidth equally important, it actually performs the power pre-compensation role in the ISBI cancelation bandwidth (e.g., the edge subbands of two services bands) to some degree, as an extra benefit.

\section{NON-SYNCHRONIZED SYSTEM AND CHANNEL EQUALIZATION ALGORITHMS IN THE PRESENCE OF TRANSCEIVER IMPERFECTIONS}

In the previous section, we focused on the ISBI performance analysis of the GS-MS-UFMC system with perfectly synchronized transceiver and sufficient ZP. However, due to the hardware impairments and imperfect synchronization mechanisms, a certain level of CFO, TO and PN will always be present in practical systems. Moreover, sufficient ZP length is not always guaranteed (and sometimes unnecessary) in order to reduce the overhead of the system. Thirdly, designing a GS system may not be practical in some scenarios. In this section, we will first derive the system model by taking all aforementioned imperfections into consideration. The system performance is analyzed in terms of power of desired signal, ICI, ISI, ISBI and noise. Finally, new one-tap equalization algorithms are proposed. Note that the following analysis and derivations are applicable to both uplink and downlink transmissions, though the channel and synchronization schemes can be different.

A. Non-synchronized MS-UFMC signal model with transceiver imperfections and insufficient $Z P$

To generalize the derivation, let us consider $\tilde{N}_{s y m, k}$ UFMC symbols for the $k$-th service. We can rewrite the $N_{k, \text { all }}=$ $\tilde{N}_{s y m, k} N_{k}$-length signal after IDFT operation as:

$$
x_{k, v}(l)=\sum_{u=0}^{Z_{k}-1} a_{k, v, n_{l}}(u) e^{\frac{j 2 \pi\left(l-\xi_{l} N_{k}\right)\left(u+\eta_{k, v}\right)}{N_{k}}},
$$

where $l=0,1, \cdots, N_{k, \text { all }}-1$ and $n_{l}=\left\lceil(l+1) / N_{k}\right\rceil$ and $\xi_{l}=\left\lfloor l / N_{k}\right\rfloor, \eta_{k, v}$ is defined in (4). The output of the subband filter of the $v$-th subband in the $k$-th service can be expressed as

$$
q_{k, v}(d)=\sum_{l=0}^{N_{k, a l l}-1} x_{k, v}(l) f_{k, v}\left(d-l+\varphi_{d} L_{k}\right),
$$

where $\varphi_{d}=\left\lfloor d / L_{k}\right\rfloor$ and $d=0,1, \cdots, L_{k, \text { all }}-1$ with $L_{k, \text { all }}=L_{k} \tilde{N}_{s y m, k}$. For asynchronous MS-UFMC system, let us define $\mathbf{t}_{k}=\left[t_{k, 1}, t_{k, 2}, \cdots, t_{k, K}\right]$ as the synchronization error between $k$-th service and other services in samples. $t_{k, s}$ means the first sample of the $k$-th service (i.e., $q_{k, v}(0)$ ) is aligned with the $t_{k, s}$-th sample in the $s$-th service. Apparently, we have $t_{k, k}=0$. For the $k$-th service, the signal at the transmitter can be written as:

$p_{k}(d)=\sum_{s=1}^{K} \sum_{g=1}^{V_{s}} \sum_{l=0}^{N_{s, a l l}-1} x_{s, g}(l) f_{s, g}\left[d-l+t_{k, s}+\varphi_{d} L_{s}\right]$,

Considering $\tau_{m}$ as timing synchronization error in samples of the $m$-th user, the $i$-th symbol of the received signal at user $m$ belonging to the $k$-th service can be expressed as:

$$
\begin{aligned}
& y_{m, i}(r)=\sum_{d=0}^{L_{k}-1} \sum_{e=-\infty}^{\infty} p_{k}\left(d+(i-1) L_{k}\right) . \\
& h_{m}\left(r-d-e L_{k}+\tau_{m}\right)+w_{m, i}(r),
\end{aligned}
$$


where $r=0,1, \cdots, L_{k}-1$. Performing $2 N_{k}$ point DFT on $y_{m, i}(r)$ and down sampling by a factor of 2 , we have

$x_{k, v, m, i}(n)=\sum_{r=0}^{L_{k}-1} y_{m, i}(r) e^{-\frac{j 2 \pi r\left(n+\eta_{k, v}+\epsilon_{k, v}\right)}{N_{k}}+j \theta_{m, n}}+\tilde{w}_{m, i}(n)(34)$ where $n=0,1, \cdots, Z_{k}-1$ and $\epsilon_{k, v}$ is the CFO at the $v$-th subband in the $k$-th service. $\theta_{m, n}$ is phase noise of the $m$-th user following the Wiener random processing as [23]

$$
\theta_{m, n+1}=\theta_{m, n}+\phi_{m, n}, \quad \text { for } \quad 0 \leq n \leq Z_{k}-1
$$

and $\phi_{m, n} \sim \mathcal{N}\left(0, \sigma_{P N, m}^{2}=4 \pi \gamma_{m} T_{s}\right)$ with $T_{s}$ being the sampling rate of the system and $\gamma_{m}$ is the $3-\mathrm{dB}$ one-side bandwidth of the PN. Without loss of generality, we set $\theta_{m, 0}=0$.

Substituting (30), (32), (33) into (34), we have the complete signal model of $i$-th symbol for the $m$-th user that belongs to the $v$-th subband of the $k$-th service as:

$x_{k, v, m, i}(n)=\sum_{r=0}^{L_{k}-1} \sum_{d=0}^{L_{k}-1} \sum_{l=0}^{N_{k, a l l}-1} \sum_{s=1}^{K} \sum_{g=1}^{V_{s}} \sum_{u=0}^{Z_{s, g}-1} \sum_{e=-\infty}^{\infty} a_{s, g, n_{l}}(u)$

$h_{m}\left(r-d-e L_{k}+\tau_{m}\right) f_{s, g}\left(d-l+(i-1) L_{k}+t_{k, s}+\varphi_{d} L_{s}\right)$. $e^{\frac{j 2 \pi\left(l-\xi_{l} N_{s}\right)\left(u+\eta_{s, g}\right)}{N_{s}}-\frac{j 2 \pi r\left(n+\eta_{k, v}+\epsilon_{k, v}\right)}{N_{k}}+j \theta_{n}}+\tilde{w}_{m, i}(n)$.

Equation (36) is a complete signal model taking the CFO, TO, PN and insufficient ZP into consideration for nonsynchronized MS-UFMC systems in multipath environments. $x_{k, v, m, i}(n)$ (for $n=0,1, \cdots, Z_{k}-1$ ) is a length $Z_{k}$ series, corresponding to $Z_{k}$ symbols transmitted to the $m$-th user in the $v$-th subband of $k$-th service in the $i$-th symbol. Conventional one tap equalization algorithms such as ZF or MMSE can be performed. In the next subsection, we will split (36) into five components, i.e., desired signal, ICI, ISI, ISBI and noise and express their powers for SINR calculation and propose channel equalization algorithms.

\section{B. Power of desired signal, ICI, ISI, ISBI and noise}

The modulated symbols $a_{k 1, v 1, n 1}(u 1)$ and $a_{k 2, v 2, n 2}(u 2)$ are uncorrelated if $[k 1, v 1, n 1, u 1] \neq[k 2, v 2, n 2, u 2]$ and also using $\mathcal{E}\left|a_{k 1, v 1, n 1}(u 1)\right|^{2}=\rho_{\text {sym }}^{2}$, we can express the power of the signal received at the $n$-th subcarrier that belongs to the $v$-th subband of the $k$-th service in terms of desired signal, ISI, ICI, ISBI and noise as follows:

$P_{x}(n)=P_{D}(n)+P_{I C I}(n)+P_{I S I}(n)+P_{I S B I}(n)+\frac{L_{k}}{N_{k}} \sigma^{2}$

where

$$
\begin{aligned}
& P_{D}(n)=\rho_{\text {sym }}^{2} \mathcal{E}|\beta(k, v, n, n, 0)|^{2}, \\
& P_{I C I}(n)=\rho_{\text {sym }}^{2} \sum_{g=1}^{V_{k}} \sum_{u=0}^{Z_{k, g}-1} \mathcal{E}|\beta(k, g, n, u, 0)|^{2} \\
& g \neq v \text { or } u \neq n, \\
& P_{I S I}(n)=\rho_{\text {sym }}^{2} \sum_{g=1}^{V_{k}} \sum_{u=0}^{Z_{k, g}-1} \sum_{e=-\infty, e \neq 0}^{\infty} \mathcal{E}|\beta(k, g, n, u, e)|^{2}, \\
& P_{I S B I}(n)=\rho_{\text {sym }}^{2} \sum_{s=1, s \neq k}^{K} \sum_{e=-\infty}^{\infty} \sum_{g=1}^{V_{s}} \sum_{u=0}^{Z_{s, g}-1} \mathcal{E}|\beta(s, g, n, u, e)|^{2}(38)
\end{aligned}
$$

and $\beta(s, g, n, u, e)$ can be expressed as:

$$
\begin{aligned}
& \beta(s, g, n, u, e)=\sum_{d=0}^{L_{k}-1} \sum_{r=0}^{L_{k}-1} \sum_{l=0}^{N_{s, a l l}-1} h_{m}\left(r-d-e L_{k}+\tau_{m}\right) \\
& e^{\frac{j 2 \pi\left(l-\xi_{l} N_{s}\right)\left(u+\eta_{s, g}\right)}{N_{s}}-\frac{j 2 \pi r\left(n+\eta_{k, v}+\epsilon_{k, v}\right)}{N_{k}}+j \theta_{n}} \\
& f_{s, g}\left(d-l+(i-1) L_{k}+t_{k, s}+\varphi_{d} L_{s}\right)
\end{aligned}
$$

To simplify the expression of $|\beta(s, g, n, u, e)|^{2}$, let us define

$T_{m, i}\left(s, g, d_{1}, d_{2}, u\right)=\tilde{T}_{m, i}\left(s, g, d_{1}, u\right) \tilde{T}_{m, i}^{*}\left(s, g, d_{2}, u\right)$,

where $\tilde{T}_{m, i}(s, g, d, u)=\sum_{l=0}^{N_{s, a l l}-1} e^{\frac{j 2 \pi\left(l-\xi_{l} N_{s}\right)\left(u+\eta_{s, g}\right)}{N_{s}}} f_{s, g}(d-$ $\left.l+(i-1) L_{k}+t_{k, s}+\varphi_{d} L_{s}\right)$. We assume that the channel vector $\mathbf{h}_{k, i}$ has the following property: $\mathcal{E}\left\{h_{m}\left(l_{1}\right) h_{m}^{*}\left(l_{2}\right)\right\}=\delta\left(l_{1}-l_{2}\right) R_{m}\left(l_{1}\right)$, where $R_{m}\left(l_{1}\right)$ is the autocorrelation function of the channel $\mathbf{h}_{m}$ at the $l_{1}$-th path and $l_{2}$-th path. $\delta(l)$ is the Kronecker delta function with $\delta(l)=1$ for $l=0$ and $\delta(l)=0$ for $l \neq 0$.

Using (35), (40) and the channel property, we have

$$
\begin{aligned}
& \mathcal{E}|\beta(s, g, n, u, e)|^{2}=\sum_{d_{1}=0}^{L_{k}-1} \sum_{d_{2}=0}^{d_{1}} T_{m, i}\left(s, g, d_{1}, d_{2}, u\right) \sum_{r=d_{1}-d_{2}}^{L_{k}-1} \\
& e^{\frac{j 2 \pi\left(r_{2}-r_{1}\right)\left(n+\eta_{k, v}\right)}{N_{k}}} R\left(r-d-e L_{k}+\tau_{m}\right) e^{-\frac{1}{2} \sigma_{P N}^{2}\left|d_{1}-d_{2}\right|} \\
& +\sum_{d_{1}=0}^{L_{k}-1} \sum_{d_{2}=d_{1}}^{L_{k}-1} T_{m, i}\left(s, g, d_{1}, d_{2}, u\right) \sum_{r=d_{1}-d_{2}}^{L_{k}-1-\left(d_{2}-d_{1}\right)} \\
& e^{\frac{j 2 \pi\left(r_{2}-r_{1}\right)\left(n+\eta_{k, v}\right)}{N_{k}}} R\left(r-d-e L_{k}+\tau_{m}\right) e^{-\frac{1}{2} \sigma_{P N}^{2}\left|d_{1}-d_{2}\right|} .(41)
\end{aligned}
$$

In the presence of interference for non-synchronized MSUFMC system, the SINR of the $n$-th subcarrier can be written as:

$$
\operatorname{SINR}(n)=\frac{P_{D}(n)}{P_{I C I}(n)+P_{I S I}(n)+P_{I S B I}(n)+\frac{\sigma^{2} L_{k}}{N_{k}}} .
$$

\section{Channel Equalization}

Based on the derived signal model in the presence of transceiver imperfections and insufficient ZP length for nonsynchronized MS-UFMC system, the channel equalization algorithms can be updated accordingly. In this paper, two most widely used linear equalizers: ZF and MMSE are considered. The equalizer for the $n$-th subcarrier can be expressed as

$$
W_{n}=\frac{\beta(k, v, n, n, 0)^{H}}{|\beta(k, v, n, n, 0)|^{2}+\nu \sigma_{e f f}(n)^{2} / \rho_{s y m}^{2}},
$$

where $\nu=0$ and $\nu=1$ correspond to ZF and MMSE equalizer, respectively. $\sigma_{\text {eff }}(n)^{2}=P_{I S I}(n)+P_{I C I}(n)+$ $P_{I S B I}(n)+\frac{L_{k}}{N_{k}} \sigma^{2}$ is the effective interference-plus-noise power for the $n$-th subcarrier of the $v$-th subband in the $k$ th service taking ISI, ICI and ISBI into consideration. Note that the channel, noise variance, the value of CFO, TO and $\mathrm{PN}$ for equalizer calculation are assumed to be known or can be estimated by receiver prior to demodulation.

The proposed one-tap channel equalization algorithm employs the desired signal structural property (i.e., $\left.\beta(k, v, n, n, 0)^{H}\right)$, and the second order statistics of the 
interference-plus-noise. Note that more sophisticated MMSEbased equalization algorithm can be applied to improve the performance by employing the interference structural property. However, this will make the algorithm much more complicated and additionally, the availability of the interference signal structural property might not be available. On the other hand, the ZF-based equalizer (i.e., $\nu=0$ ) in (43) does not use the interference-plus-noise information.

The ISBI cancelation algorithm proposed in Section III is for the GS system. While the equalizer and the analysis proposed in (43) are for a non-synchronized system, which is more general. However, in this case, the ISBI cancelation algorithm might be not (practically) applicable since the symbol overlapping in a such system is irregular.

\section{NUMERICAL RESUlts}

In this section, we use Monte-Carlo simulations to verify the accuracy of derived GS-MS-UFMC system signal models and Propositions proposed in (12), (15) and (16). In addition, the ISBI cancelation algorithms proposed in (27) will be examined in the first set of simulations with perfect transceivers. In the presence of transceiver imperfections for the non-synchronized MS-UFMC system, the system model and derived desired signal, ICI, ISI and ISBI in equation (38) will be verified in the second set of simulations. Finally, we will investigate the proposed channel equalization algorithms in (43) and combine with the proposed ISBI cancelation algorithms in (27) to compare with OFDM and SoTA UFMC systems in terms of BER.

The signal is modulated using 64-QAM with power normalized to unity and the input SNR is controlled by the noise variance. We adopt FIR Chebyshev filter [5] with $50 \mathrm{~dB}$ side lobe attenuation. For fair comparison, we assume the guard interval for OFDM system is the same as the total overhead for UFMC system for all simulations. We also provide the results for OFDM systems as benchmark for comparison.

\section{A. ISBI in GS-MS-UFMC systems}

In order to examine the ISBI in MS systems, we first consider AWGN channel without CP/ZP inserting in both OFDM and UFMC systems. However, these assumptions will be void in the next set of simulations in order to demonstrate the system performance in practical scenarios. We only give analytical results first. The analytical results will be compared with the simulation results later for more general case. Note that ISBI is only generated and affected by adjacent subbands belonging to two different services. We consider two type of services and each has one subband only, and both subbands contain 20 subcarriers. The filter length $\mathbf{L}_{F, \text { equal }}=[5,10]$ for two subbands, respectively, in MS-UFMC systems.

Let us first investigate the impact of different subcarrier spacing between two subbands on the level of ISBI. We set $B_{1}=0.2$ and vary $B_{2}$ in the range of $0.2,0.1,0.08,0.05,0.025,0.0125$. Then the two subband subcarrier spacings have the following relationships: $M_{s c}=$ $\Delta f_{1} / \Delta f_{2}=1,2,2.5,4,8$, respectively. The guard band is set as $\Delta B_{1}=\Delta f_{1}$ and $\Delta B_{0}=0.2$. Fig. 4 shows that ISBI in the second subband periodically becomes zero when $M \in \mathbb{Z}^{+}$ (note index 21 is the first subcarrier of subband 2), which verifies Proposition 2. In addition, when $M_{s c}$ increases, number of subcarriers in subband 2 with non-zero ISBI increases which leads to average performance loss. On the other hand, when $M_{s c}$ is non-integral (e.g. $M_{s c}=2.5$ ), as shown in Fig. 4, ISBI at all of the subcarriers is non-zero. Moreover, the ISBI value at any given subcarrier is at relatively higher level. Therefore, to reduce ISBI, $M_{s c}$ is preferred to be an integer number. On the other hand, we can see the interference at subband 1 has the opposite trend. With $M_{s c}$ increasing, the ISBI values in subband 1 reduce, although the change is not as significant as in subband 2 .

To examine the impact of GB on the performance in terms of ISBI, we fix $M_{s c}=2$ and vary $\Delta B_{1}$ such that $\Delta B_{1}=$ $0, \Delta f_{2}, 1.2 \Delta f_{2}, 2 \Delta f_{2}, 4 \Delta f_{2}, 8 \Delta f_{2}$, i.e., $M_{g b}=\Delta B_{1} / \Delta f_{2}=$ $0,1,1.2,2,4,8$, respectively, as shown in Fig. 5. Again, zero ISBI values occur periodically in subband 2 for integral $M_{g b}$, which is in line with Proposition 2. Additionally, the nonzero ISBI value at any subcarrier index reduces significantly with increased guard band. When $M_{g b}=1.2$, non-zero ISBI is observed at all subcarriers, and the maximum values (or peak values) of ISBI are significantly reduced compared with integral $M_{g b}$. This provides us a hint for frame structure design for multi-service systems: one can select non-integral subcarrier spacing as GB to reduce the peak ISBI values. For subband 1, ISBI values sharply reduce when the index of subcarrier reduces from index 20 (i.e., last subcarrier of subband 1).

In order to show the worst case of ISBI, we assume the minimum output SINR (with noise power $=-50 \mathrm{~dB}$ in order to make the system interference limited) among the subcarriers for both subbands by varying GB. The results for both UFMC and OFDM systems are shown in Fig. 6, where it is observed that when GB increases, minimum SINR increases accordingly. For UFMC system, the worst SINR case in subband 2 shows linear relationship with GB. In subband 1, where the minimum SINR tends to attain a steady value, for large GB for all $M_{s c}$ and both OFDM and UFMC systems. Fig. 6 also shows that with subband filtering, the UFMC system can outperform OFDM system in terms of the worst case SINR in all cases.

To demonstrate the effectiveness of the proposed ISBI cancelation algorithm in (27), the MSE of the symbol estimation versus subcarrier index is shown in Fig. 7 for different bandwidths for ISBI cancelation, $N_{c a n}=N_{c a n, 1}=N_{c a n, 2}=$ $4,8,20$ subcarrier. Note that $N_{c a n}=20$ means that all subcarriers in the subbands are considered. Since the ZF and MMSE algorithms show similar trend, we only give more practical and simpler ZF-based results. As shown in Fig. 7, when $N_{c a n}=4$, ISBI values at the subcarrier indices 17 , $18,19,20$ for the first subband, and 21, 22, 23, 24 for the second, drop compared to the original algorithm without ISBI cancelation algorithm and this reduction is more significant when $N_{c a n}=8$. When all of the subcarriers are taken into consideration for interference cancelation, we can see that the MSE at all subcarriers can reach noise power level, whereas some subcarriers have even lower MSE values than the noise 


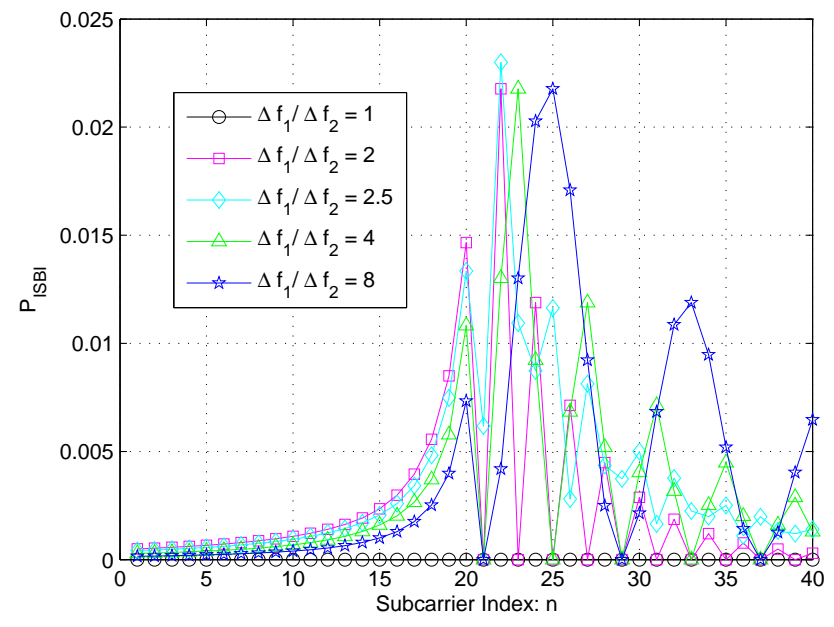

Fig. 4. ISBI versus subcarrier index for different subcarrier spacing $\Delta f_{1} / \Delta f_{2}$ in GS-MS-UFMC systems.

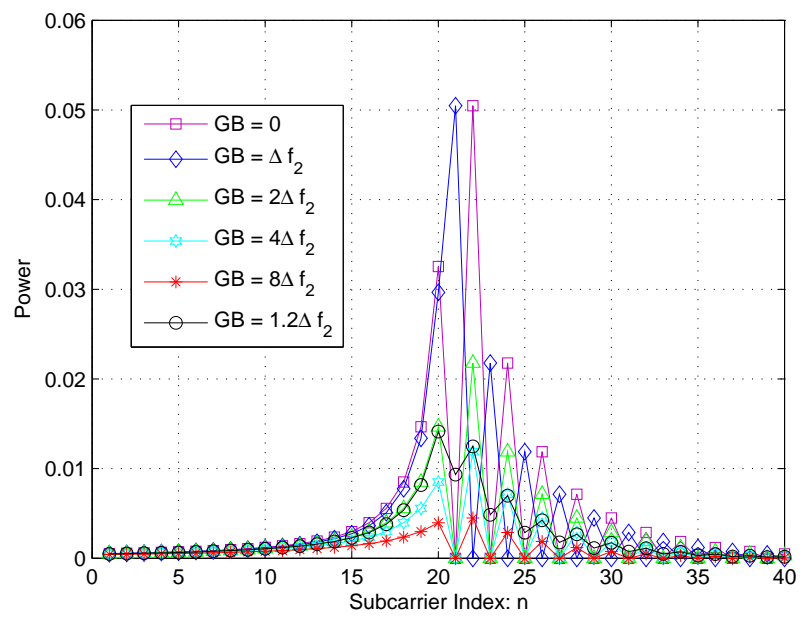

Fig. 5. ISBI versus subcarrier index for different guard band $\Delta B_{1}$.

power since the power allocated to subcarriers is uneven in UFMC systems.

\section{B. Generalized signal model verification}

Here, we examine the effects of different system parameters on the system performance in more practical scenarios and compare our analytical results with the simulation results in terms of the power of the desired signal, ICI, ISI and ISBI in a non-synchronized system. We consider moderate ISBI with $B_{1}=0.2, B_{2}=0.1$ and $\Delta B_{1}=4 \Delta f_{1}$. The generalized synchronization error is set as $\mathbf{t}_{1,2}=[0,0.3]$ and $\mathbf{t}_{2,1}=[0.6,0]$. The receivers of the two users are assumed to have different values of CFO, with $\epsilon=\left[\epsilon_{1}, \epsilon_{2}\right]=[0.01,0.005]$, and the TO values are set as $\boldsymbol{\tau}=\left[\tau_{1}, \tau_{2}\right]=[0.02,0.02]$. We use Wiener random processing to model $\mathrm{PN}$ [23] with sampling duration $=3.2 \mathrm{~ns}$, and $3 \mathrm{~dB}$ bandwidth PN $\gamma_{1}=\gamma_{2}=50 \mathrm{~Hz}$, which correspond to PN variance $\sigma_{P N, 1}^{2}=\sigma_{P N, 2}^{2}=0.0002$ for both

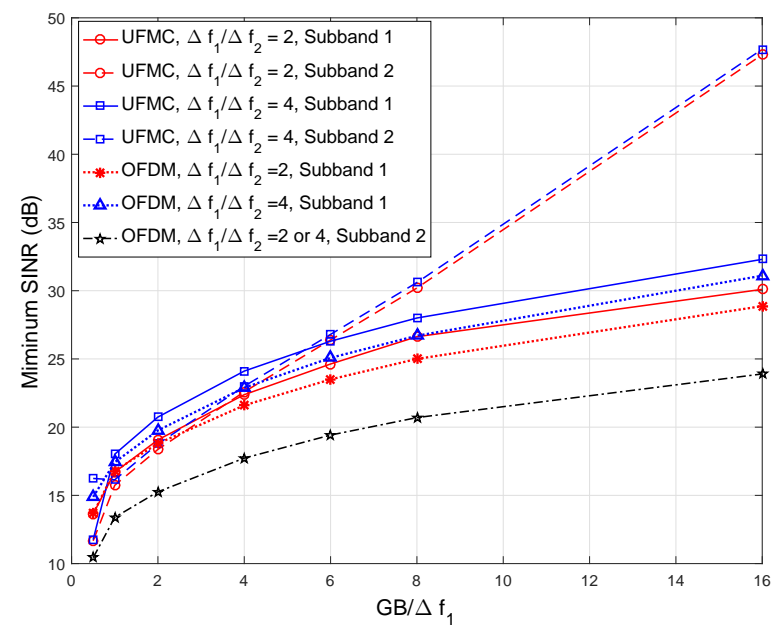

Fig. 6. Minimum output SINR (worst case) versus guard band $\Delta B_{1}$ for both GS-MS-OFDM and GS-MS-UFMC systems.

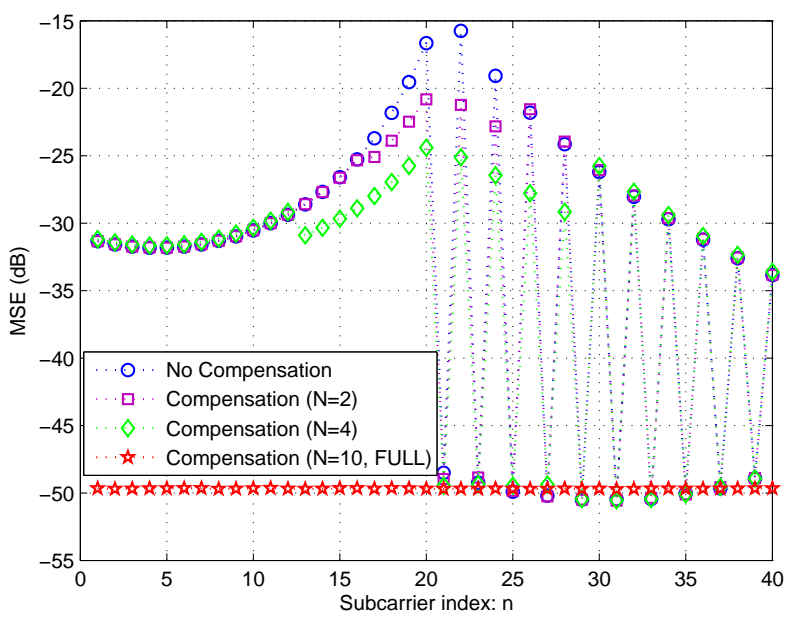

Fig. 7. Information symbol estimation MSE versus subcarrier index for both with and without and proposed ISBI cancelation algorithms in GS-MS-UFMC systems $\left(\Delta f_{1} / \Delta f_{2}=2, \Delta B_{1}=\Delta f_{1}\right)$.

services. We consider the channels for the two users are LTE EVA and ETU channels, respectively.

Analytical results for desired signal $P_{D}(n)$ derived in equation (38) are compared with simulation results and shown in Fig. 8. It can be seen that all of the analytical results match the simulation results which shows the effectiveness and accuracy of the derived signal models. The UFMC system shows frequency selectivity over each subband, while the OFDM system shows equal response at all subcarriers.

The analytical results for ICI, ISI and ISBI power in equation (38) are compared with simulation results for both UFMC and OFDM systems in Fig. 9. Again, the analytical results match simulated results in all cases. As shown in Fig. 9, the maximum ISBI power is in the two adjacent subcarriers of the two subbands (i.e., subcarrier indices 20 and 21). The ICI and ISI, however, show lower interference level in this 


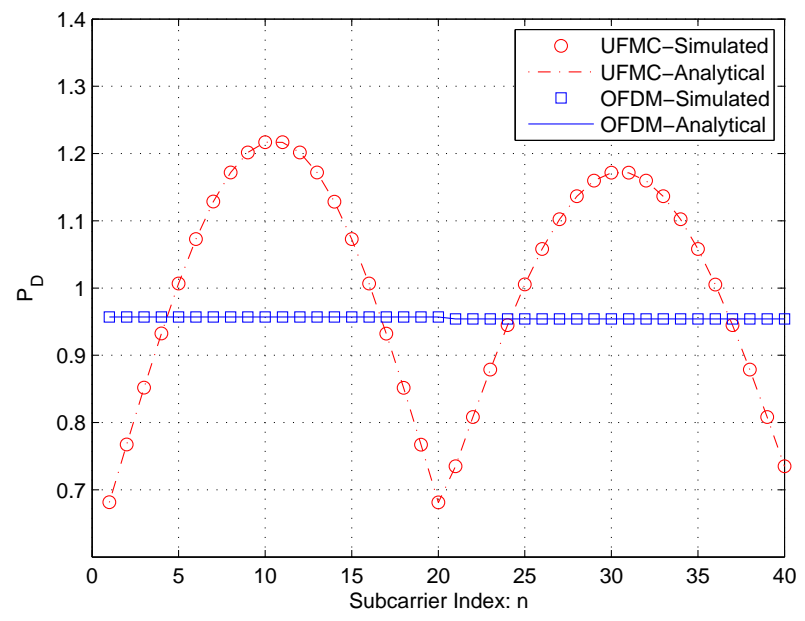

Fig. 8. Power of desired signal in difference subcarriers for non-synchronized MS-UFMC and MS-OFDM system in the presence of transceiver imperfection

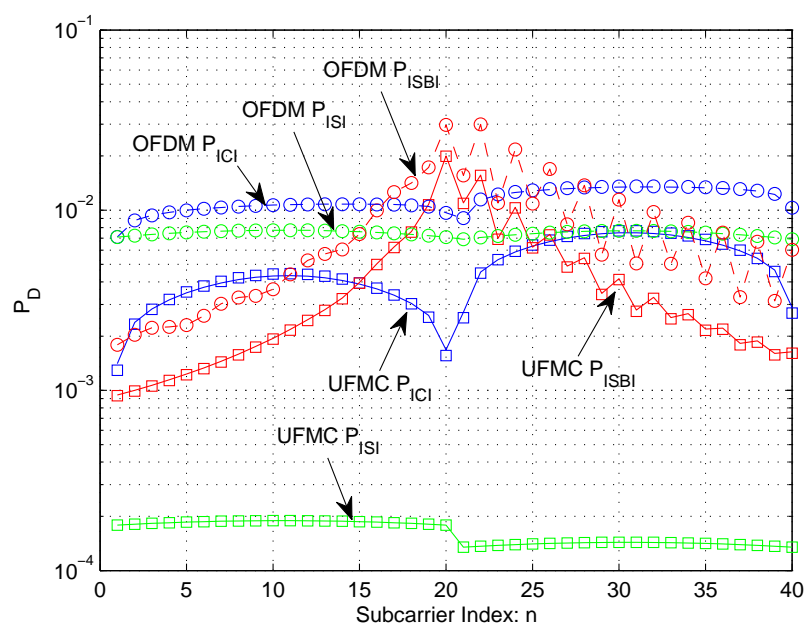

Fig. 9. Power of ICI, ISI and ISBI in difference subcarriers for non-synchronized MS-UFMC and MS-OFDM system in the presence of transceiver imperfection (lines: Analytical results, circles and squares: simulated results).

region since the filtering response has a lower gain at the edge of subband than in the middle (see Fig. 8), leading to reduced interference at the edges than in the middle of a subband. However, even with moderate ISBI, it is more dominant in the neighboring subcarriers of two adjacent bands, which will become evident later. In addition, there is non-zero ISBI at all subcarriers for both UFMC and OFDM systems, even if the subcarriers index satisfies Proposition 2, since we have considered a non-synchronized system.

Finally, we examine the effectiveness of the proposed ISBI cancelation algorithm and equalization algorithm given in equation (27) and (43) in the presence of CFO, TO and PN. Since the proposed ISBI cancelation algorithms are applicable only to generalized synchronized system, simulations are conducted in this scenario. The results are compared with the

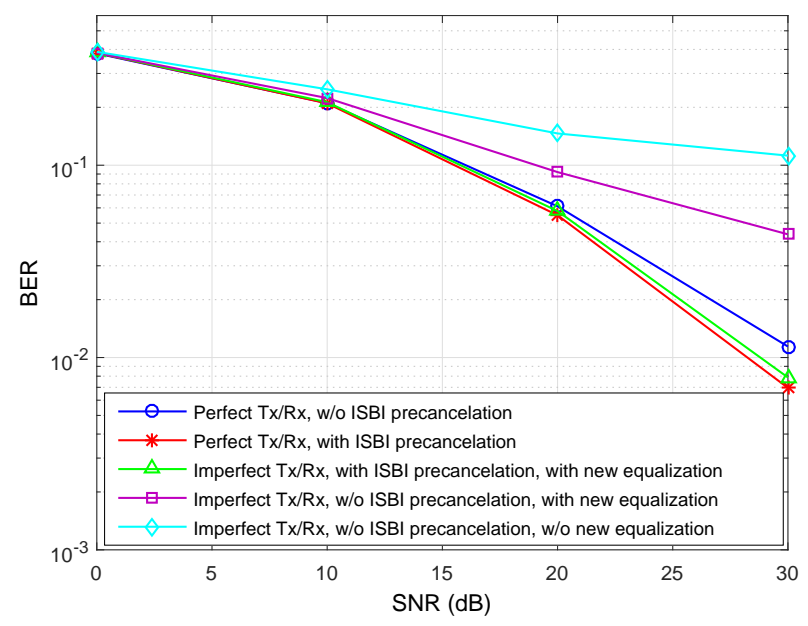

Fig. 10. BER performance versus input SNR.

interference-free case (i.e., perfect $\mathrm{Tx} / \mathrm{Rx}$ ) serving as benchmark. We only present results for the MMSE-based channel equalization algorithm and the ZF-based ISBI precancelation algorithms, but it must be noted that the MMSE-based ISBI precancelation and ZF-based equalizers show similar trend. The simulation settings are the same as in the last simulation. In the interference-free case, the proposed ISBI cancelation algorithm can effectively improve the system performance in terms of BER. In the presence of CFO, TO and PN, the proposed channel equalization algorithm can significantly reduce the BER for given input SNR compared with the system using original channel equalization. The performance gain is even larger when both proposed ISBI precancelation and channel equalization algorithms are adopted in the imperfect transceiver case, which verifies that proposed joint transceiver algorithm works effectively.

\section{CONClusions}

A framework for multi-service subband filtered multi-carrier systems has been established in this paper. We first classified different implementations of MS-SFMC system in both time and frequency domains to provide a valid frame structure design methodology for next generation wireless communications and beyond. The system model is proposed by dividing the whole bandwidth into a number of subbands each for a different type of service. We focused on the ISBI performance analysis and a number of properties and observations were provided to shed light on the frame structure design. Based on the proposed model, the low-complexity ISBI cancelation algorithms were proposed at the transmitter that can flexibly cancel the ISBI for arbitrary bandwidth. By considering the transceiver imperfections (CFO, TO and $\mathrm{PN}$ ), synchronization errors among services and insufficient $\mathrm{ZP}$ length, an analytical system model was established to analyze the desired signal, ISI, ICI, ISBI and noise. Consequently, channel equalization algorithms were proposed by considering the errors and imperfections based on the derived signal models. All of the derivations and propositions are also applicable to OFDM 
systems as a special case. Various numerical and simulation results are given to show the effectiveness of the analysis and proposed algorithms.

\section{ACKNOWLEDGEMENT}

The authors would like to acknowledge the support of the University of Surrey 5GIC (http://www.surrey.ac.uk/5gic) members for this work.

\section{APPENDIX A \\ PROOF OF EQUATION (10)}

Using (6), (7) then we can write $\mathbf{c}_{m, i}=\left(\tilde{\mathbf{D}}_{k, v}^{H} \tilde{\mathbf{y}}_{m, i}\right)^{\downarrow 2}$ as

$\mathbf{c}_{m, i}=\frac{1}{\rho_{k, v}}\left(\tilde{\mathbf{D}}_{k, v}^{H} \tilde{\mathbf{B}}_{m} \mathbf{G}_{k, v, i} \mathbf{a}_{k, v}\right)^{\downarrow 2}+\left(\tilde{\mathbf{D}}_{k, v}^{H} \mathbf{w}_{m, i}\right)^{\downarrow 2}$

$+\frac{1}{\rho_{k, g}}\left(\tilde{\mathbf{D}}_{k, v}^{H} \tilde{\mathbf{B}}_{m} \sum_{g=1, g \neq v}^{V_{k}} \mathbf{G}_{k, g, i} \mathbf{a}_{k, g}\right)^{\downarrow 2}+\frac{1}{\rho_{n, l}}\left[\sum_{n=1, n \neq k}^{K} \sum_{l=1}^{V_{n}}\right.$

$\left.\tilde{\mathbf{D}}_{k, v}^{H}\left(\tilde{\mathbf{B}}_{m} \mathbf{G}_{n, l, i}+\boldsymbol{\Delta} \mathbf{B}_{m} \mathbf{G}_{n, l-1, i}\right) \mathbf{a}_{n, l}\right]^{\downarrow 2}$,

where $\tilde{\mathbf{B}}_{m}=\left[\mathbf{B}_{m} ; \mathbf{0}_{\left(N_{k}-L_{C H, m}-L_{F, k}+2\right) \times L_{k}}\right]$. Note that the subcarriers in the subbands that belong to one type of service are orthogonal when guard interval is sufficient [22], leading to ICI term $\left(\tilde{\mathbf{D}}_{k, v}^{H} \tilde{\mathbf{B}}_{m} \sum_{g=1, g \neq v}^{V_{k}} \mathbf{G}_{k, g, i} \mathbf{a}_{k, g}\right)^{\downarrow 2}=\mathbf{0}$. Using $\mathbf{D}_{2 N_{k}} \mathbf{D}_{2 N_{k}}^{H}=\mathbf{I}_{2 N_{k}}$, (44) can be further expressed as

$$
\begin{aligned}
& \mathbf{c}_{m, i}=\left[\tilde { \mathbf { D } } _ { k , v } ^ { H } \mathbf { B } _ { m , c i r } \mathbf { D } _ { 2 N _ { k } } \left(\frac{1}{\rho_{k, v}} \mathbf{D}_{2 N_{k}}^{H} \tilde{\mathbf{G}}_{k, v, i} \mathbf{a}_{k, v}\right.\right. \\
& \left.+\frac{1}{\rho_{n, l}} \mathbf{D}_{2 N_{k}}^{H} \sum_{n=1, n \neq k}^{K} \sum_{l=1}^{V_{n}} \tilde{\mathbf{G}}_{n, l, i} \mathbf{a}_{n, l}\right)+\tilde{\mathbf{D}}_{k, v}^{H} \mathbf{w}_{m, i} \\
& \left.\left.+\frac{1}{\rho_{n, l}} \sum_{n=1, n \neq k}^{K} \sum_{l=1}^{V_{n}} \tilde{\mathbf{D}}_{k, v}^{H} \mathbf{\Delta} \mathbf{B}_{m} \mathbf{G}_{n, l, i-1}\right) \mathbf{a}_{n, l}\right]^{\downarrow 2},
\end{aligned}
$$

where $\mathbf{B}_{m, \text { cir }}$ is a $2 N_{k}$ dimension circular channel matrix with the first column $\left[\mathbf{h}_{m}^{T} ; \mathbf{0}_{\left(2 N_{k}-L_{C H, m}\right) \times 1}\right]$. When $n=k$ and $l=v$, according to the definition of $\mathbf{G}_{k, v}$ in (7), we can write $\mathbf{D}_{2 N_{k}}^{H} \tilde{\mathbf{G}}_{k, v, i} \mathbf{a}_{k, v}=\mathbf{D}_{2 N_{k}}^{H} \mathbf{A}_{k, v, c i r} \mathbf{D}_{2 N_{k}} \mathbf{D}_{2 N_{k}}^{H} \tilde{\mathbf{D}}_{k, v} \mathbf{a}_{k, v, i}$, where $\tilde{\mathbf{D}}_{k, v}=\left[\mathbf{D}_{k, v} ; \mathbf{0}_{N_{k} \times Z_{k}}\right]$ and $\mathbf{A}_{k, v, c i r}$ being circular matrix with the first column $\left[\mathbf{f}_{k, v}^{T} ; \mathbf{0}_{\left(2 N_{k}-L_{F, k}\right) \times 1}\right]$. By using the circular matrix property, we have $\tilde{\mathbf{D}}_{k, v}^{H} \mathbf{B}_{m, c i r} \mathbf{D}_{2 N_{k}}=$ $\tilde{\mathbf{H}}_{m, v}$ and $\mathbf{D}_{2 N_{k}}^{H} \mathbf{A}_{k, v, c i r} \mathbf{D}_{2 N_{k}}=\mathbf{F}_{k, v, a l l}$, where $\tilde{\mathbf{H}}_{m, v}=$ $\left[\ddot{\mathbf{H}}_{m, v}, \mathbf{0}_{\left(2 N_{k}-2 Z_{k}\right) \times 2 Z_{k}}\right]$ and $\ddot{\mathbf{H}}_{m, v} \in \mathbb{C}^{2 Z_{k} \times 2 Z_{k}}$ and $\mathbf{F}_{k, v, \text { all }} \in \mathbb{C}^{2 N_{k} \times 2 N_{k}}$ are diagonal matrices corresponding to the frequency domain channel and filter response. By using $\tilde{\mathbf{H}}_{m, v} \mathbf{F}_{k, v, a l l}=\ddot{\mathbf{H}}_{m, v} \ddot{\mathbf{F}}_{m, v}$ with their $l$-th diagonal element being $\ddot{H}_{m, i}(l)=\sum_{n=0}^{L_{C H, k}-1} h_{m}(n) e^{j 2 \pi n\left(l+2 \eta_{k, v}\right) /\left(2 N_{k}\right)}$ and $\ddot{F}_{k, v}(l)=\sum_{n=0}^{L_{F, k}-1} f_{k, v}(n) e^{j 2 \pi n\left(l+2 \eta_{k, v}\right) /\left(2 N_{k}\right)}$, in addition, $\left(\mathbf{D}_{2 N_{k}}^{H} \tilde{\tilde{\mathbf{D}}}_{k, v} \mathbf{a}_{k, v, i}\right)^{\downarrow 2}=\mathbf{a}_{k, v, i}$, we have the first item being $\frac{1}{\rho_{k, v}} \mathbf{H}_{m, v} \mathbf{F}_{k, v} \mathbf{a}_{k, v, i}$.

Noting that $\tilde{\mathbf{H}}_{m, v} \mathbf{D}_{2 N_{k}}^{H}=\ddot{\mathbf{H}}_{m, v} \tilde{\mathbf{D}}_{k, v}^{H}$. Then the second term in (45) can be expressed as $\frac{1}{\rho_{n, l}} \mathbf{H}_{m, v} \sum_{n=1, n \neq k}^{K} \sum_{l=1}^{V_{n}} \hat{\mathbf{D}}_{k, v}^{H} \tilde{\mathbf{G}}_{n, l, i} \mathbf{a}_{n, l} \quad$ with $\left(\tilde{\mathbf{D}}_{k, v}^{H}\right)^{\downarrow 2}=\hat{\mathbf{D}}_{k, v}^{H}$.

For the third term on noise, noting the definition of $\tilde{\mathbf{D}}_{k, v}^{H}$ and $\tilde{\mathbf{w}}_{m, i}$, it can be easily shown that $\left(\tilde{\mathbf{D}}_{k, v}^{H} \mathbf{w}_{m, i}\right)^{\downarrow 2}=\tilde{\mathbf{w}}_{m, i}$.
For the last term, we have $\left(\tilde{\mathbf{D}}_{k, v}^{H} \mathbf{\Delta} \mathbf{B}_{m} \mathbf{G}_{n, l, i-1} \mathbf{a}_{n, l}\right)^{\downarrow 2}=$ $\hat{\mathbf{D}}_{k, v}^{H} \boldsymbol{\Delta} \mathbf{B}_{m} \mathbf{G}_{n, l, i-1} \mathbf{a}_{n, l}$. Substituting the three derived terms into (45), we derive (12).

\section{APPENDIX B}

\section{PROOF OF PROPOSITION 1 AND PROPOSITION 2}

Since $\Delta f_{1}=M_{s c} \Delta f_{2}$, the symbol for service 2 overlaps $M_{s c}$ independent UFMC symbols in service 1, e.g., we have $N_{\text {sym }, 1}=M_{s c}$ and $N_{s y m, 2}=1$. According to equation (11), ISBI for the first subband (also the only subband) in service 2 can be written as

$$
\mathbf{y}_{2,1, I S B I}=\frac{1}{\rho_{1,1}} \mathbf{H}_{2,1} \hat{\mathbf{D}}_{2,1}^{H} \hat{\mathbf{G}}_{1,1} \mathbf{a}_{1}
$$

where $\hat{\mathbf{G}}_{1,1}=\left[\mathbf{G}_{1,1} ; \mathbf{0}_{\left(2 N_{2}-M L_{1}\right) \times M_{s c} Z_{1}}\right]$. With sufficient ZP, we have utilized the fact $\hat{\mathbf{D}}_{2,1}^{H} \mathbf{\Delta} \mathbf{B}_{2,1} \mathbf{G}_{1,1, i-1} \mathbf{a}_{1,1}=\mathbf{0}$. Since $\mathbf{G}_{1,1}=\operatorname{Blkdiag}\left(\mathbf{A}_{1,1} \mathbf{D}_{1,1}, N_{\text {sym }, 1}\right)=\sum_{i=1}^{M_{s c}} \hat{\mathbf{A}}_{1,1, i} \mathbf{D}_{1,1, \text { all }}$ with $\hat{\mathbf{A}}_{1,1, i}=\left[\mathbf{0}_{(i-1) L_{1} \times M_{s c} Z_{1}} ; \mathbf{A}_{1, i} ; \mathbf{0}_{\left(M_{s c}-i\right) L_{1} \times M_{s c} Z_{1}}\right.$;

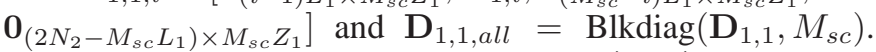
Substitute into (46), we have $\hat{\mathbf{D}}_{2,1}^{H} \hat{\mathbf{G}}_{1,1} \mathbf{a}_{1}=$ $\sum_{i=1}^{M_{s c}} \hat{\mathbf{F}}_{1, i} \mathbf{D}_{2 N_{2}}^{H} \hat{\mathbf{D}}_{1,1, \text { all }} \mathbf{a}_{1}$, where $\quad \hat{\mathbf{D}}_{1,1}=$ $\left[\mathbf{D}_{1,1, a l l} ; \mathbf{0}_{N_{2} \times Z_{1} M_{s c}}\right] . \hat{\mathbf{F}}_{1, i}=\left[\boldsymbol{\Lambda}_{1, i} \tilde{\mathbf{F}}_{1,1}, \mathbf{0}_{\left(2 N_{2}-Z_{1}\right) \times 2 N_{2}}\right]$ with $\boldsymbol{\Lambda}_{1, i}$ being $Z_{1}$ dimension diagonal matrix with its $l$-th element being $e^{-j 2 \pi l(i-1) L_{1} /\left(2 N_{2}\right)} \cdot \tilde{\mathbf{F}}_{1,1}$ is also a diagonal matrix with its $l$-th diagonal element being $\tilde{F}_{1}(l)$. Then $\sum_{i=1}^{M_{s c}} \hat{\mathbf{F}}_{1, i} \mathbf{D}_{2 N_{2}}^{H} \hat{\mathbf{D}}_{1,1, \text { all }} \mathbf{a}_{1}=\sum_{i=1}^{M_{s c}} \hat{\mathbf{F}}_{1, i} \tilde{\mathbf{D}}_{1,1}^{H} \hat{\mathbf{D}}_{1,1, \text { all }} \mathbf{a}_{1}$ with $\tilde{\mathbf{D}}_{1,1}^{H}$ being the first $Z_{1}$ columns of $\left(\mathbf{D}_{2 N_{2}}^{H}\right)^{\downarrow 2}$. Note that the $l$-th row and $n$-th column element of $\hat{\mathbf{D}}_{2 N_{2}}^{H}$ can be written as $e^{j 2 \pi\left(l+\left(B_{1}+\Delta B_{1}\right) L_{1}\right) n /\left(M_{s c} N_{1}\right)}$, by further noticing $\tilde{\mathbf{D}}_{1,1, \text { all }}$ is a block diagonal matrix, the $l$-th row and $k$-th column of $\tilde{\mathbf{D}}_{1,1}^{H} \hat{\mathbf{D}}_{1,1, \text { all }}$ is $\varpi_{l, k}=$ $\sum_{n=0}^{N_{1}-1} e^{-j 2 \pi n k / N_{1}} e^{j 2 \pi\left(l+\left(B_{1}+\Delta B_{1}\right) N_{1}\right)\left(n+\kappa N_{1}\right) /\left(M_{s c} N_{1}\right)}=$ $\left\lfloor\left(k / Z_{1}\right\rfloor\right.$ Therefore, the ISBI to the $l$-th subcarrier in the subband 2 can obtain Proposition 1.

By using $\Delta B_{1}=M_{g b} \Delta f_{2}$, we can further express $\varpi_{l, k}=\sum_{n=0}^{N_{1}-1} e^{-j 2 \pi n\left[\frac{k-l / M_{s c}-\left(M_{1}+M_{g b} / M_{s c}\right)}{N_{1}}\right]}$. Apparently, when $l / M_{s c}+M_{g b} / M_{s c} \in \mathbb{Z}$ and $k-l / M_{s c}-\left(M_{1}+\right.$ $\left.M_{g b} / M_{s c}\right) \neq 0, \sum_{n=0}^{N_{1}-1} e^{-j 2 \pi n\left[\frac{k}{N_{1}}-\frac{l+\left(B_{1}+\Delta B_{1}\right) N_{1}}{M_{s c} N_{1}}\right]}=0$ for all $k$. Note for non-overlapping bandwidth allocation, we have $k-l / M_{s c}-\left(M_{1}+M_{g b} / M_{s c}\right)<0$ for all $k$. i.e, the only condition to achieve $P_{I S B I, 2}(l)=0$ is $l / M_{s c}+M_{g b} / M_{s c} \in \mathbb{Z}$, which implies that $l+M_{g b}$ is integral times of $M_{s c}$, resulting in Proposition 2.

\section{APPENDIX C \\ PROOF OF PROPOSITION 3}

According to (12), the ISBI for the $i$-th UFMC symbol in subband 1 of a service can be written as

$$
\mathbf{y}_{1,1, i, I S B I}=\frac{1}{\rho_{2,1}} \mathbf{H}_{1,1} \hat{\mathbf{D}}_{1,1}^{H} \hat{\mathbf{G}}_{2,1, i} \mathbf{a}_{2,1}
$$

using $\hat{\mathbf{D}}_{1,1}^{H} \hat{\mathbf{G}}_{2,1, i} \mathbf{a}_{2,1}=\hat{\mathbf{D}}_{1,1}^{H} \hat{\mathbf{A}}_{2,1, i} \mathbf{D}_{2,1} \mathbf{a}_{2,1}$, where $\hat{\mathbf{A}}_{2,1, i}$ denotes taking the $\left[(i-1) L_{1}+1\right]$-th to $i L_{1}$-th row of $\mathbf{A}_{2,1}$. For $L_{F, 2}<<L_{1}$, we can use the approximation $\hat{\mathbf{A}}_{2,1, i} \tilde{\mathbf{D}}_{2,1} \approx$ $\mathbf{A}_{2,1, \text { cir }} \hat{\mathbf{D}}_{2,1, i}$, where $\mathbf{A}_{2,1, \text { cir }} \in \mathbb{C}^{2 N_{1} \times 2 N_{1}}$ is a circular matrix with the first column being $\left[\mathbf{f}_{2,1}, \mathbf{0}_{1 \times\left(2 N_{1}-L_{F, 2}\right)}\right]^{T}$ and 
$\hat{\mathbf{D}}_{2,1, i}=\left[\mathbf{0}_{(i-1) L_{1} \times Z_{2}} ; \ddot{\mathbf{D}}_{2,1, i} ; \mathbf{0}_{\left(2 N_{1}-i L_{1}\right) \times Z_{2}}\right]$, where $\ddot{\mathbf{D}}_{2,1, i}^{H}$ is a sub-matrix of $\mathbf{D}_{2,1}$ taking its $\left[(i-1) L_{1}+1\right]$-th to the $i L_{1}$-th rows. Thus, we have $\hat{\mathbf{D}}_{1,1}^{H} \mathbf{A}_{2,1, c i r} \hat{\mathbf{D}}_{2,1, i} \mathbf{a}_{2,1}=$ $\tilde{\mathbf{F}}_{2} \hat{\mathbf{D}}_{1,1}^{H} \hat{\mathbf{D}}_{2,1, i} \mathbf{a}_{2,1}$, with the $l$-th diagonal element of $\tilde{\mathbf{F}}_{2}$ being $\tilde{F}_{2,1}=\sum_{n=0}^{L_{F, 2}-1} e^{-j 2 \pi n l / N_{1}} f_{1,1}(n)$. The $l$-th row and the $k$-th column of the matrix $\hat{\mathbf{D}}_{1,1}^{H} \hat{\mathbf{D}}_{2,1, i}$ can be written as $\varpi_{l, k, i}=\sum_{n=0}^{L_{1}-1} e^{-j 2 \pi\left[M_{s c} l n-\left(n+(i-1) L_{1}\right)\left(k+\eta_{2,1}\right)\right] /\left(M_{s c} N_{1}\right)}$. Then we get Proposition 3 .

\section{REFERENCES}

[1] J. Andrews, S. Buzzi, W. Choi, S. Hanly, A. Lozano, A. Soong, and J. Zhang, "What will 5G be?" IEEE Journal on Selected Areas in Communications, vol. 32, no. 6, pp. 1065-1082, June 2014.

[2] Cisco, "Cisco visual networking index: Global mobile data traffic forecast update, 2015-2020," Tech. Rep., 2016.

[3] A. Ijaz, L. Zhang, M. Grau, A. Mohamed, et al., "Enabling massive IoT in 5G and beyond systems: PHY radio frame design considerations," IEEE Access, vol. 4, pp. 3322 - 3339, 2016.

[4] X. Zhang, M. Jia, L. Chen, J. Ma, and J. Qiu, "Filtered-OFDM - enabler for flexible waveform in the 5th generation cellular networks," in IEEE Globecom, 2015, pp. 1-6.

[5] 5GNOW, "D3.2: 5G waveform candidate selection," Tech. Rep., 2014.

[6] G. Wunder, P. Jung, M. Kasparick, et al., "5GNOW: non-orthogonal, asynchronous waveforms for future mobile applications," IEEE Communications Magazine, vol. 52, no. 2, pp. 97-105, February 2014.

[7] V. Vakilian, T. Wild, F. Schaich, S. Ten Brink, and J.-F. Frigon, "Universal-filtered multi-carrier technique for wireless systems beyond LTE," in IEEE Globecom Workshops (GC Wkshps), 2013, pp. 223-228.

[8] Y. Chen, F. Schaich, and T. Wild, "Multiple access and waveforms for 5G: IDMA and universal filtered multi-carrier," in IEEE Vehicular Technology Conference (VTC Spring), 2014, pp. 1-5.

[9] F. Schaich and T. Wild, "Waveform contenders for 5G; OFDM vs. FBMC vs. UFMC," in International Symposium on Communications, Control and Signal Processing (ISCCSP), May 2014, pp. 457-460.

[10] J. Abdoli, M. Jia, and J. Ma, "Filtered OFDM: A new waveform for future wireless systems," in IEEE Signal Processing Advances in Wireless Communications (SPAWC), June 2015, pp. 66-70.

[11] B. Farhang-Boroujeny, "OFDM versus filter bank multicarrier," IEEE Signal Processing Magazine, vol. 28, no. 3, pp. 92-112, May 2011.

[12] J. Du, P. Xiao, J. Wu, and Q. Chen, "Design of isotropic orthogonal transform algorithm-based multicarrier systems with blind channel estimation," IET Communications, vol. 6, pp. 2695-2704, November 2012.

[13] G. Fettweis, M. Krondorf, and S. Bittner, "GFDM - generalized frequency division multiplexing," in IEEE Vehicular Technology Conference, 2009, April 2009, pp. 1-4.

[14] N. Michailow, M. Matthe, I. Gaspar, A. Caldevilla, et al., "Generalized frequency division multiplexing for 5th generation cellular networks," IEEE Transactions on Communications, vol. 62, no. 9, pp. 3045-3061, Sept 2014

[15] E. Dahlman, S. Parkvall, and J. Skold, 4G: LTE/LTE-Advanced for Mobile Broadband. Academic Press, 2011.

[16] D. S. Sesia, M. M. Baker, and M. I. Toufik, LTE: The UMTS Long Term Evolution: from Theory to Practice, 2nd ed. Wiley-Blackwell, 2011.

[17] X. Wang, T. Wild, F. Schaich, and A. Fonseca dos Santos, "Universal filtered multi-carrier with leakage-based filter optimization," in European Wireless Conference, 2014, pp. 1-5.

[18] J. Yli-Kaakinen and M. Renfors, "Optimization of flexible filter banks based on fast-convolution," in IEEE International Conference on Acoustics, Speech and Signal Processing (ICASSP), 2014, pp. 8317-8320.

[19] M. Renfors, J. Yli-Kaakinen, and F. J. Harris, "Analysis and design of efficient and flexible fast-convolution based multirate filter banks," IEEE Transactions on Signal Processing, vol. 62, no. 15, pp. 3768-3783, 2014

[20] M. Renfors, J. Yli-Kaakinen, T. Levanen, M. Valkama, et al., "Efficient fast-convolution implementation of filtered CP-OFDM waveform processing for 5G," in IEEE Globecom Workshops (GC Wkshps), 2015, pp. $1-7$.

[21] X. Wang, T. Wild, and F. Schaich, "Filter optimization for carrierfrequency-and timing-offset in universal filtered multi-carrier systems," in IEEE Vehicular Technology Conference (VTC Spring), 2015, pp. 1-6.

[22] L. Zhang, A. Ijaz, P. Xiao, et al., "MU-UFMC system performance analysis and optimal filter length and zero padding length design," submitted to IEEE Transactions on Signal Processing, 2016. [Online]. Available: http://arxiv.org/pdf/1603.09169v1.pdf
[23] R. Hamila, O. Ozdemir, and N. AL-Dhahir, "Beamforming OFDM performance under joint phase noise and I/Q imbalance," IEEE Transactions on Vehicular Technology, vol. PP, no. 99, pp. 1-1, 2015. 